\title{
Circular RNA circRNF13 inhibits proliferation and metastasis of nasopharyngeal carcinoma via SUMO2
}

Yongzhen $\mathrm{Mo}^{1,2+}$, Yumin Wang ${ }^{2,3 \dagger}$, Shuai Zhang ${ }^{3}$, Fang Xiong ${ }^{4}$, Qijia Yan ${ }^{3}$, Xianjie Jiang ${ }^{1,2}$, Xiangying Deng ${ }^{1,2}$, Yian Wang 1,2, Chunmei Fan 1,2, Le Tang 1,2, Shanshan Zhang ${ }^{4}$, Zhaojian Gong5, Fuyan Wang', Qianjin Liao', Can Guo ${ }^{2}$, Yong Li ${ }^{6}$, Xiaoling $\mathrm{Li}^{1,2}$, Guiyuan $\mathrm{Li}^{1,2}$, Zhaoyang Zeng ${ }^{1,2^{*}}$ (ID and Wei Xiong ${ }^{1,2^{*}}$ (D)

\begin{abstract}
Background: Circular RNAs (circRNAs) are widely expressed in human cells and are closely associated with cancer development. However, they have rarely been investigated in the context of nasopharyngeal carcinoma (NPC).

Methods: We screened a new circRNA, circRNF13, in NPC cells using next-generation sequencing of mRNA. Reverse transcription polymerase chain reaction and RNA fluorescence in situ hybridization were used to detect circRNF13 expression in 12 non-tumor nasopharyngeal epithelial (NPE) tissues and 36 NPC samples. Cell proliferation was detected using MTT and flow cytometry assays, and colony formation capability was detected using colony formation assays. Cell migration and invasion were analyzed using wound-healing and Transwell assays, respectively. Cell glycolysis was analyzed using the Seahorse glycolytic stress test. Glucose transporter type 1 (GLUT1) ubiquitination and SUMOylation modifications were analyzed using co-immunoprecipitation and western blotting. CircRNF13 and Small Ubiquitin-like Modifier 2 (SUMO2) interactions were analyzed using RNA pull-down and luciferase reporter assays. Finally, to test whether circRNF13 inhibited NPC proliferation and metastasis in vivo, we used a xenograft nude mouse model generated by means of subcutaneous or tail vein injection.
\end{abstract}

Results: We found that circRNF13 was stably expressed at low levels in NPC clinical tissues and NPC cells. In vitro and in vivo experiments showed that circRNF13 inhibited NPC proliferation and metastasis. Moreover, circRNF13 activated the SUMO2 protein by binding to the $3^{\prime}$ - Untranslated Region (3'-UTR) of the SUMO2 gene and prolonging the halflife of SUMO2 mRNA. Upregulation of SUMO2 promotes GLUT1 degradation through SUMOylation and ubiquitination of GLUT1, which regulates the AMPK-mTOR pathway by inhibiting glycolysis, ultimately resulting in the proliferation and metastasis of NPC.

Conclusions: Our results revealed that a novel circRNF13 plays an important role in the development of NPC through the cirCRNF13-SUMO2-GLUT1 axis. This study implies that circRNF13 mediates glycolysis in NPC by binding to SUMO2 and provides an important theoretical basis for further elucidating the pathogenesis of NPC and targeted therapy.

Keywords: Nasopharyngeal carcinoma, circRNF13, SUMO2, GLUT1, Glycolysis, Proliferation, Metastasis

\footnotetext{
*Correspondence: zengzhaoyang@csu.edu.cn; xiongwei@csu.edu.cn †Yongzhen Mo and Yumin Wang contributed equally to this work.

${ }^{1}$ NHC Key Laboratory of Carcinogenesis and Hunan Key Laboratory of Cancer Metabolism, Hunan Cancer Hospital and Affiliated Cancer Hospital of Xiangya School of Medicine, Central South University, Changsha 410078, Hunan, China

Full list of author information is available at the end of the article
}

\section{Background}

Nasopharyngeal carcinoma (NPC) is a malignant tumor of epithelial origin in the nasopharynx, with an incidence rate of up to 2 in 1,000 in Southern China and Southeast Asia [1-4]. Because NPC occurs in the nasopharynx, its 
early symptoms are not obvious, but NPC have a strong tendency to invade and metastasize. Clinical studies show that approximately $70 \%$ of patients with NPC experience metastasis to the lymph nodes in the neck, and almost all patients in advanced stages have invasive growth to the skull base [5-7]. However, to date, the molecular mechanisms involved in the development of NPC, especially in the metastatic process, are still unclear. Therefore, further understanding of the pathogenesis of NPC is needed to develop more effective therapeutic strategies to combat this disease.

Circular RNAs (circRNAs) are a newly discovered class of noncoding RNAs in recent years, and the study of their biological function and relationship with diseases has become a frontier of biomedical research [8-11]. CircRNAs are a group of RNAs with a loop-like structure that lacks a polyA tail. They are not easily degraded by RNA enzymes and have very stable properties due to their special loop structure [12-15]. With the development of the next-generation sequencing technology in recent years, an increasing number of circRNAs have been discovered, and more than 30,000 circRNAs have currently been identified in the transcriptome of various human cell types [16, 17]. An increasing number of studies have found that circRNAs have important functions in numerous life processes and are involved in the development of many human diseases, including malignant tumors $[18,19]$. Although many circRNAs have been identified, the functions of the vast majority of circRNAs are still unclear, especially in NPC.

Intracellular protein ubiquitination is an important post-translational modification that is widely found in eukaryotic cells. The vast majority of intracellular proteins are degraded via the ubiquitin-dependent proteasome pathway. Small ubiquitin-like modifier 2 (SUMO2) is a member of the SUMO family, and SUMOylation is a type of ubiquitin-like protein modification. SUMOylation and ubiquitination often occur at the same lysine residues of a substrate protein, and SUMOylation can sometimes antagonize ubiquitination in the regulation of transcription factors [20]. SUMOylation can also promote the ubiquitination and degradation of modified cytoplasmic, mitochondrial, and membrane proteins, serving as a signal for the recruitment of ubiquitin E3 ligases in various biological processes [21].

Glucose transporter proteins (GLUTs) are one of the most important transmembrane proteins in the body that are responsible for glucose transport and reabsorption in different tissues and organs of the body. High expression of GLUT1 in various tumors, such as liver, gastric, and breast cancers, is involved in the regulation of malignant phenotypes, such as tumor metastasis and proliferation $[22,23]$. It provides favorable conditions for glycolysis through massive glucose uptake, providing more energy and synthetic raw materials for tumor cells. In addition, the large amount of lactic acid produced by glycolysis alters the microenvironment of tumor cells, which is more favorable for tumor cell invasion and metastasis.

In this study, we identified a novel circRNA molecule, circRNF13 (circBase ID: has_circ_0001346), which is expressed at low levels in NPC. The proliferation and metastasis of circRNF13 were assessed in NPC cells through in vitro and in vivo experiments. Further studies revealed that $\operatorname{circRNF13}$ directly binds and stabilizes SUMO2 mRNA, which upregulates the protein levels of SUMO2 and promotes GLUT1 degradation by means of SUMOylation and ubiquitination, thus inhibiting the glycolytic process and proliferation, migration, and invasion of NPC.

\section{Methods \\ NPC clinical samples}

In total, 12 non-tumor nasopharyngeal epithelial (NPE) tissues and 36 NPC samples were collected at the Affiliated Cancer Hospital of Central South University (Changsha, China). This study was approved by the Joint Ethics Committee of the Central South University Health Authority, and informed consent was obtained from all participants. Diagnoses of all specimens were confirmed via histopathological examination.

\section{Cell culture, plasmids, and transfection}

NPC cell lines 5-8F, HNE2, CNE2, HONE1, and 6-10B and immortalized normal nasopharyngeal epithelial NP69 cells were obtained from the Cell Center of Central South University. All cells were cultured in RPMI-1640 medium (Gibco) supplemented with 10\% fetal bovine serum (Gibco). All cells were maintained at $37{ }^{\circ} \mathrm{C}$ in a humidified incubator with $5 \% \mathrm{CO}_{2}$.

The circRNA expression plasmid pCirc was generously provided by Prof. Li Yong at Baylor College of Medicine for RNA circulation. For circRNF13 overexpression, cDNAs reverse-transcribed from RNAs of CNE2 cells were used as PCR templates and subjected to amplification of the full-length circRNF13. The reporter plasmid pMIR-Luciferase-Reporter for SUMO2 and inserts of the SUMO2 3'UTR sequence within the pMIR-Luciferase-Reporter plasmid were constructed with the One Step Cloning Kit (Vazyme). Plasmids were transfected into cells using Lipofectamine 3000 Reagent (Invitrogen, USA). Primers used are listed in Table S1.

\section{RNA isolation and RT-PCR}

Total RNA was extracted using TRIzol reagent (Life Technologies) and subjected to reverse transcription with random primers using the $5 \times$ All-In-One kit (Abm). 
Then, the expression levels of target RNAs were measured with SYBR Green Master Mix using a StepOnePlus Real-Time PCR System (Applied Biosystems). $\beta$-actin was used as an endogenous control, and the fold change was calculated via the $2^{-\Delta \Delta C T}$ method. Primers used are listed in Table S1.

\section{Cytosolic/nuclear fraction assay}

Cells were resuspended in hypotonic buffer $(25 \mathrm{mM}$ Tris- $\mathrm{HCl}, \mathrm{pH} 7.4,1 \mathrm{mM} \mathrm{MgCl}, 5 \mathrm{mM} \mathrm{KCl}$ ) and incubated on ice for $5 \mathrm{~min}$ before adding an equal volume of hypotonic buffer containing $1 \%$ NP-40 for an additional $5 \mathrm{~min}$. After centrifuging the cells at $5,000 \mathrm{~g}$ for $5 \mathrm{~min}$, the supernatant was collected as the cytosolic fraction. Pellets were washed twice with hypotonic buffer and then resuspended in nuclear resuspension buffer $(20 \mathrm{mM}$ HEPES, pH 7.9, $400 \mathrm{mM} \mathrm{NaCl}, 1 \mathrm{mM}$ EDTA, $1 \mathrm{mM}$ EGTA, $1 \mathrm{mM}$ DTT, $1 \mathrm{mM}$ PMSF). After incubation on ice for $30 \mathrm{~min}$, samples were centrifuged at 12,000 $\mathrm{g}$ for $10 \mathrm{~min}$, and supernatants were collected as the nuclear fraction. Reverse transcription of RNA was used for RTPCR detection. Primers used are listed in Table S1.

\section{RNase $\mathrm{R}$ treatment}

RNase $R$ treatment experiments were performed to verify the stability of circRNA. Five micrograms of total RNA was extracted from NPC cells and divided into two groups. One group was incubated with $1 \mu \mathrm{l}$ RNase $\mathrm{R}\left(20 \mathrm{U} / \mu \mathrm{l}\right.$; Epicenter, WI, USA) at $37^{\circ} \mathrm{C}$ for $30 \mathrm{~min}$, and the other group was treated without RNase R. RNA was incubated at $70{ }^{\circ} \mathrm{C}$ for $10 \mathrm{~min}$ to inactivate RNase R and then reverse-transcribed for RT-PCR detection. Primers used are listed in Table S1.

\section{Fluorescence in situ hybridization (FISH)}

A digoxigenin-labeled specific probe for $\operatorname{circRNF13}$ was designed and synthesized by Sangon. The FISH experiment was performed according to the manufacturer's instructions (GenePharma). First, cells were fixed and permeabilized using $0.25 \%$ Triton X-100. Then, cells were hybridized with specific probes overnight at $37{ }^{\circ} \mathrm{C}$ with circRNF13 in hybridization buffer. Nuclei were counterstained with DAPI (Invitrogen, D1306, USA). Cells were imaged using a confocal microscope (Ultra-View Vox, Perkin-Elmer, Waltham, MA, USA). The circRNF13 probe used is listed in Table S1.

\section{MTT and cell cycle assays}

For MTT assays, 1,000 cells per well were seeded into 96-well plates for the MTT assay. Cells were incubated with $0.5 \mathrm{mg} / \mathrm{mL}$ filtered sterile MTT (Beyotime) at $37^{\circ} \mathrm{C}$ for $4 \mathrm{~h}$ at the indicated time point. Then, the media were removed and replaced with $200 \mu \mathrm{L}$ DMSO, and absorbance was measured at $490 \mathrm{~nm}$.

Cell cycle assays were performed using flow cytometry according to the manufacturer's guidelines. To examine the cell cycle, measurements were performed. Briefly, cells were trypsinized, collected and stained in solution with RNase or PI for $15 \mathrm{~min}$ at $25{ }^{\circ} \mathrm{C}$. Flow cytometry analysis was immediately performed using a FACSCalibur.

\section{Colony formation assay}

For colony formation assay. In brief, 2,000 cells were plated into 6-well plates after transfected circRNF13 overexpression vector or $\operatorname{circRNF13}$ siRNA for $48 \mathrm{~h}$. The cells were allowed to grow for the next 7 days to allow colony formation and the colonies were visualized using crystal violet staining.

\section{Wound healing assay}

Cells were cultured in 6-well plates for $24 \mathrm{~h}$ and then wounded using a sterilized pipet tip to make a straight scratch. After gentle washing with D-Hanks, cells were cultured in RPMI-1640 medium with 1\% FBS. Pictures were taken using an Olympus digital camera at 0 and $24 \mathrm{~h}$ after wounding (at least three randomly selected fields were imaged).

\section{Transwell assays}

Cell invasion assays were performed using a Transwell chamber (Millipore). RPMI-1640 medium supplemented with $20 \%$ FBS was added to the bottom chambers, and then cells were suspended in RPMI-1640 medium and seeded into the top chamber, which was coated with Matrigel. After incubating at $37^{\circ} \mathrm{C}$ for $48 \mathrm{~h}$, cells that did not migrate through the pores were removed using a cotton swab. The Transwell chambers were fixed in $4 \%$ paraformaldehyde for $30 \mathrm{~min}$, followed by staining with $1 \%$ crystal violet for $10 \mathrm{~min}$. Cells on the bottom of the chamber were counted using an inverted phase-contrast microscope (at least three randomly selected fields were quantified).

\section{Animal experiments}

We purchased 4-week-old female BAL B/c nude mice from the Experimental Animal Center of Central South University (Changsha, China) and raised them in an SPF-free barrier environment. For lung metastasis experiments, nude mice were randomly divided into four groups ( $n=7$ per group). Each nude mouse was injected via the tail vein with $2 \times 10^{6}$ NPC CNE2 cells transfected with the circRNF13 overexpression vector, $\operatorname{circRNF13}$ siRNA, the blank plasmid, or scrambled siRNA. After eight weeks, nude mice were sacrificed by 


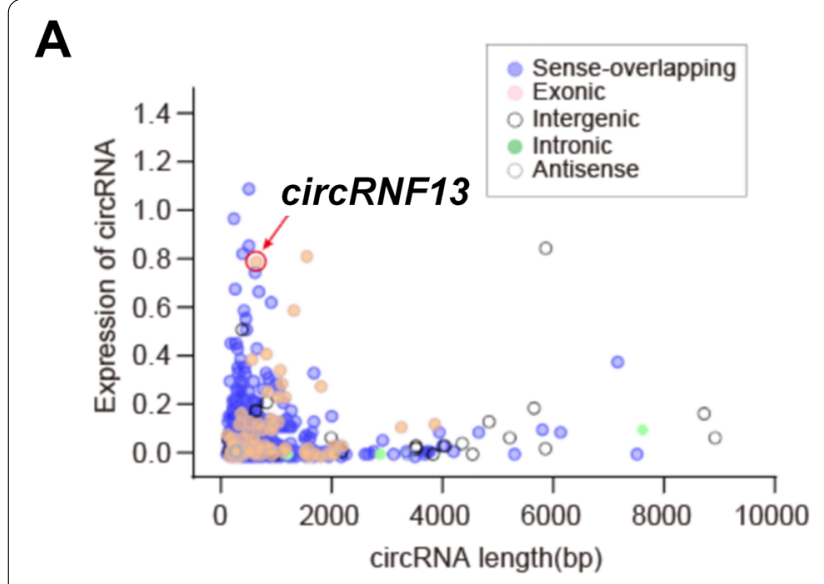

B

C

RNF13
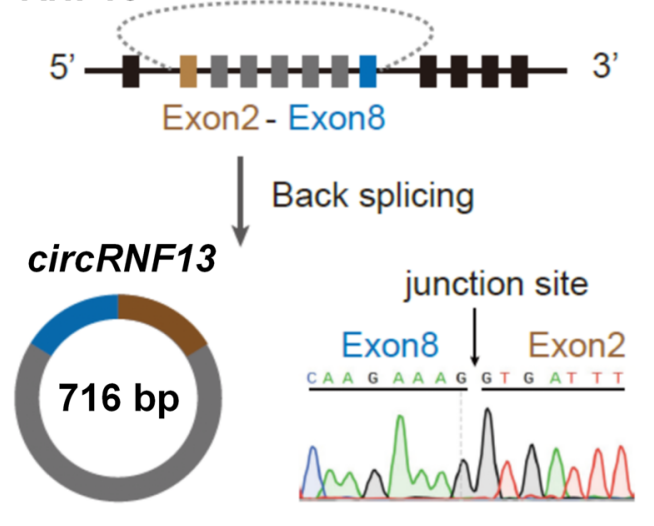

D

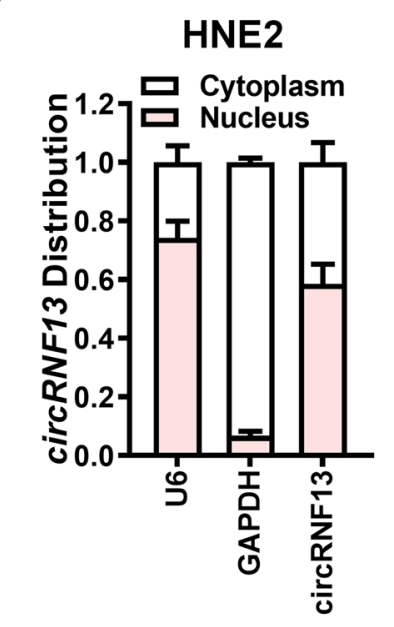

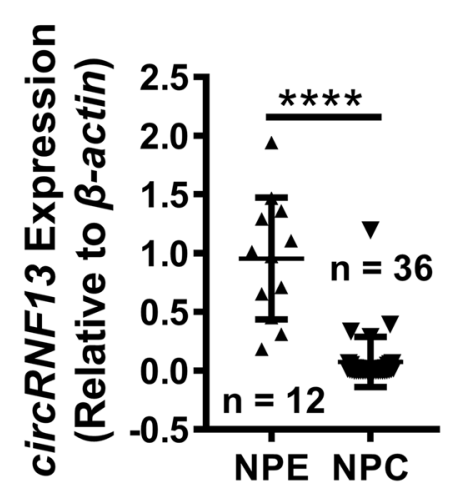

E

$\mathbf{F}$
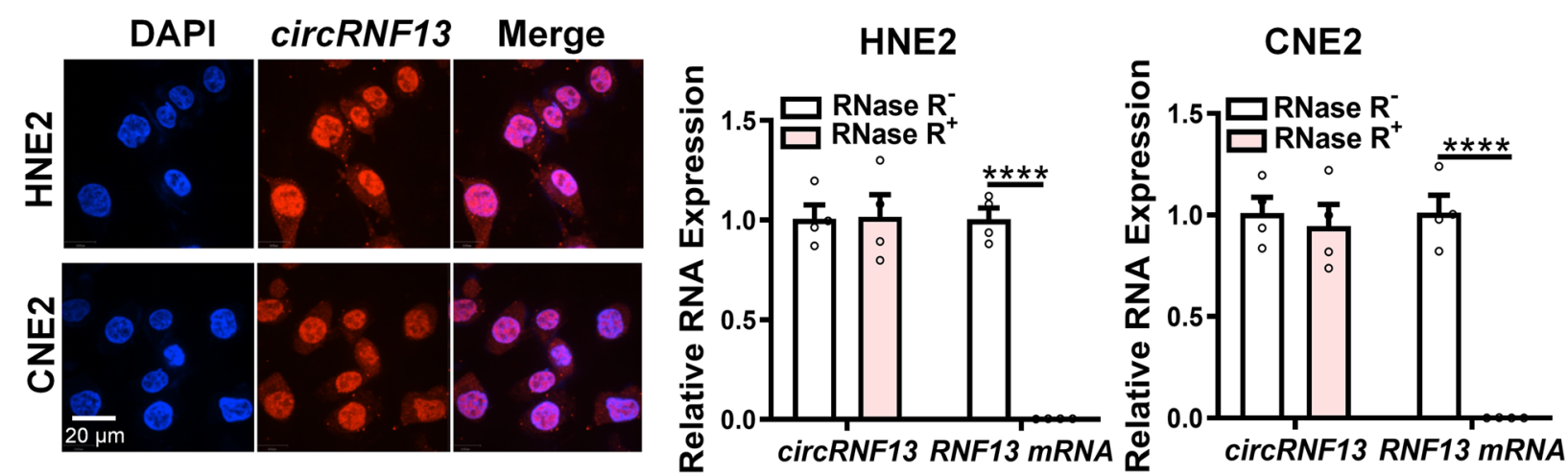

Fig. 1 CircRNF13 expression is lowly expressed in NPC. A Expression profile of circular RNAs (circRNAs) in 5-8F cells. Five types were included: exon-derived circRNAs, intron-derived circRNAs, anti-sense strand circRNAs, overlapping regions of sense strand circRNAs, and intergenic circRNAs, according to their different looping regions. CircRNF13 was ranked 14th among the top 20, and was screened due to its high abundance (RPM = 0.59). B Expression of circRNF13 was examined in NPC $(n=36)$ and NPE $(n=12)$ tissues using RT-PCR, NPE, non-tumor nasopharyngeal epithelial tissues. Data have been represented as mean \pm standard deviation (SD). ${ }^{* * *}, p<0.0001$. C Schematic structure of circRNF13. circRNF13 is 716 bp in length and circularly spliced by 2-8 exons of the RNF13 gene (RefSeq: NM_183383.2) on the chromosome 3q25.1 region, as verified using Sanger sequencing. D circRNF13 is primarily localized in the cytoplasm, as identified using nucleoplasmic separation experiments. U6 was selected as a nuclear marker and GAPDH was selected as a cytoplasmic marker. E Intracellular localization of circRNF13 was examined using fluorescence in situ hybridization. Scale bar: $20 \mu \mathrm{m}$. F Stability of circRNF13 and RNF13 mRNA was detected in RNase R-treated HNE2 and CNE2 cells using RT-PCR. Data have been represented as mean \pm SD. ${ }^{* * *}, p<0.0001$ 
cervical dislocation. Lung tissue was removed, weighed, and imaged, and the number of nodules on the surface of the lung was recorded to assess tumor metastasis. Lung tissues were then subjected to gradient dehydration, sectioned, embedded in paraffin, and stained with $H \& E$ for histological examination.

For subcutaneous tumorigenesis experiments, nude mice were randomly divided into four groups $(n=7)$. Each nude mouse was subcutaneously injected with $3 \times 10^{6}$ NPC CNE2 cells transfected with the circRNF13 overexpression vector, circRNF13 siRNA, or the blank plasmid, scrambled siRNA. Tumor growth was monitored every 3 days. Tumor size was assessed by measuring the largest perpendicular diameters, and tumor volume was calculated as follows: $\mathrm{V}=1 / 2 \times($ length $) \times$ (width) $\times($ width). Twenty-one days after subcutaneous inoculation, mice were euthanized by cervical dislocation, and the tumor tissue was excised. The formed tumor masses were removed and weighed. All animal protocols were approved by the Institutional Laboratory of Animal Care and Use Committee at Central South University.

\section{Hematoxylin-Eosin staining (H\&E)}

Paraffin mouse tissue sections were first heated at $65{ }^{\circ} \mathrm{C}$ for $2 \mathrm{~h}$. After paraffin sections were dewaxed and hydrated, nuclei were stained with hematoxylin solution (Biosharp, Anhui, China), and then cytoplasmic staining was performed with eosin staining solution (Biosharp, Anhui, China). After the slices were dried, the sheets were preserved with neutral resin (SCR, Shanghai, China).

\section{Seahorse assays}

Assays were performed using the Seahorse XFp analyzer (Seahorse Bioscience, Agilent) according to the manufacturer's instructions. Briefly, 8000 cells/well were seeded into an 8-well XF cell culture microplate in growth medium $24 \mathrm{~h}$ before the assay. The extracellular acidification rate (ECAR) was measured using an XFp analyzer in $\mathrm{XF}$ base medium $(\mathrm{pH}=7.4)$ containing $1 \mathrm{mM}$ glutamine following sequential additions of glucose $(10 \mathrm{mM})$, oligomycin $(1.5 \mu \mathrm{M})$ and 2-DG $(50 \mathrm{mM})$. Data were analyzed by the Seahorse XF Glycolysis Stress Test Report Generator package.

\section{Liquid chromatography-mass spectrometry (LC-MS/MS)}

Mass spectrometry assays according to the manufacturer's protocol with minor modifications. Briefly, Scrambled siRNA or circRNF13 siRNA was transfected into CNE2 cells for $48 \mathrm{~h}$. Total protein was extracted and digested with protease overnight. The digested peptide mixture was dried and treated with $0.1 \%$ trifluoroacetic acid (TFA). After diluting the $5 \mu \mathrm{L}$ samples, we used an LTQ Orbitrap Velos Pro mass spectrometer (Thermo Scientific, Bremen, Germany) coupled with an Ultimate 3000 RSLC Nano System (Dionex, CA, USA) to recover proteins and perform proteomic analysis of total proteins, which were identified using Proteome Discoverer 1.4 software (Thermo Fisher Scientific, MA, USA), and the resulting original file was imported into the UniProt $\mathrm{KB} /$ Swiss-Prot database for searching. For the database search, the mass tolerances of the precursor and fragmentation were set to $10 \mathrm{ppm}$ and $0.8 \mathrm{Da}$, respectively. Peptides with a false discovery rate $<1 \%$ (q value $<0.01$ ) and proteins with an area value lower than $1 \times 10^{6}$ were discarded. Proteins that met the following criteria were considered to be differentially expressed proteins: $\geq 2$ peptides and $\geq 95 \%$ confidence; and an average fold change in protein levels $\geq 2.00$ or $\leq 0.50$. (Student's t-test, $p<0.05$ ).

\section{Enrichment analysis}

The differentially expressed proteins screened by LCMS/MS were imported to Ingenuity Pathway Analysis (IPA) software, and canonical pathways of differentially expressed genes were analyzed to obtain enrichment pathways. Fisher's exact test was used to calculate a $p$-value to determine the probability of each biological function.

\section{RNA pull-down}

The biotin-labeled circRNF13 probe was synthesized by Sangon Biotech, and the RNA pull-down assay was performed as previously described with minor modifications.

\footnotetext{
(See figure on next page.)

Fig. 2 CircRNF13 inhibits the proliferation, migration, and invasion of NPC. A Overexpression of circRNF13 inhibited, while knockdown of circRNF13 promoted proliferation of HNE2 and CNE2 cells, as assessed using MTT assay. Data have been represented as mean \pm SD. ${ }^{*}, p<0.05 ;{ }^{* *}, p<0.05 ;{ }^{* * *}$ $p<0.001$. B Clone formation assay showed that overexpression of circRNF13 inhibited the colony formation capability of HNE2 and CNE2 cells. Data have been represented as mean \pm SD. ${ }^{* * *}, p<0.001$. C CircRNF13's function in the tumor cell cycle was assessed using flow cytometry. HNE2 and CNE2 cells were stained with PI, after overexpression or knockdown of circRNF13. D CircRNF13 inhibited the migration ability of HNE2 and CNE2 cells transfected with sicircRNF13 or circRNF13 overexpression vector, as assessed using wound-healing assay. Images were acquired at 0 and 24 h. Data have been represented as mean \pm SD. ${ }^{*}, p<0.05 ;{ }^{* * *}, p<0.001$. E CircRNF13 inhibited the invasive ability of HNE2 and CNE2 cells after knockdown or overexpression of cirCRNF13, as assessed using Transwell invasion assays. Images were acquired at $24 \mathrm{~h}$, and are representative of three independent experiments. Data have been represented as mean \pm SD. ${ }^{* *}, p<0.01 ;{ }^{* *}, p<0.001$
} 


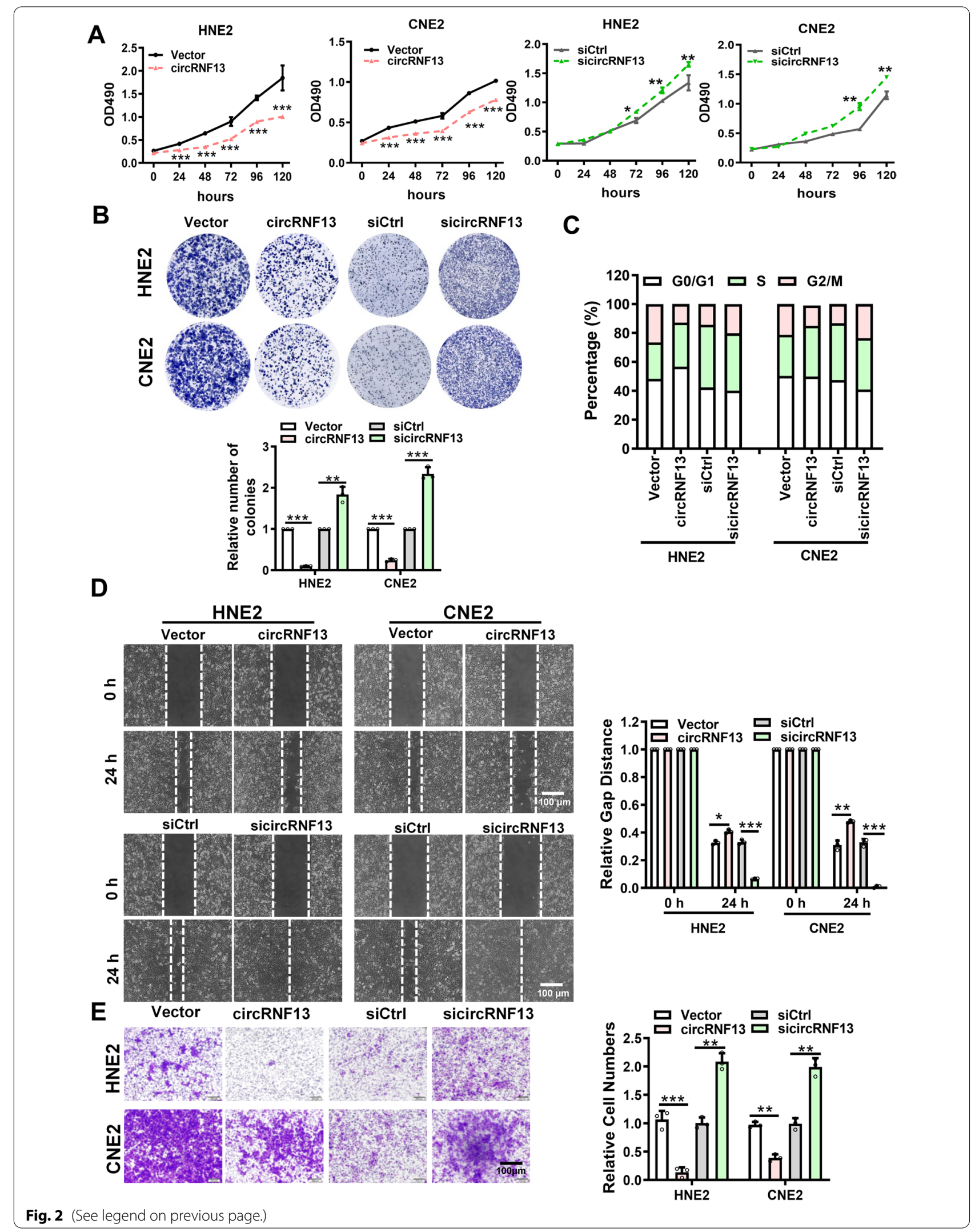


CircRNF13-overexpressing HNE2 and CNE2 cells were fixed in $1 \%$ formaldehyde for $10 \mathrm{~min}$, lysed, and sonicated. After centrifugation, $50 \mu \mathrm{L}$ of the supernatant was retained as input, and the remaining part was incubated with a circRNF13-specific probe streptavidin Dynabeads (M-280; Invitrogen) mixture overnight at $4{ }^{\circ} \mathrm{C}$. The next day, an M-280 Dynabeads probe-circRNA mixture was washed and incubated with $200 \mathrm{uL}$ of lysis buffer. Finally, TRIzol was added to the mixture for RNA extraction and detection. The circRNF13 probe and primers used are listed in Table S1.

\section{Dual-luciferase reporter assay}

The Dual-Luciferase ${ }^{\circledR}$ Reporter Assay System was used according to the manufacturer's instructions (Promega). To evaluate the interaction between circRNF13 and SUMO2, circRNF13 and pMIR-Luciferase-ReporterSUMO23'UTR plasmids were transfected in HNE2 and CNE2 cells. Forty-eight hours later, firefly and Renilla luciferase activity was examined by the Dual-Luciferase Reporter Assay System, and Renilla activity was used to normalize firefly activity.

\section{In situ hybridization (ISH)}

The ISH kit was purchased from Boster Biological (CA, USA). Paraffin mouse tissue sections were deparaffinized and rehydrated with gradient alcohol-water solution, and endogenous peroxidase was inactivated with $3 \% \mathrm{H}_{2} \mathrm{O}_{2}$. An appropriate amount of pepsin $(1 \mathrm{~mL}$ $3 \%$ citric acid solution and two drops of concentrated pepsin) was added to tissue specimens and digested at $37{ }^{\circ} \mathrm{C}$ for $15 \mathrm{~min}$. The digestion was then quickly terminated with $0.1 \mathrm{~mol} / \mathrm{L}$ glycine solution. After refixation with $4 \%$ paraformaldehyde, the prehybridization solution was used at $37^{\circ} \mathrm{C}$ for $30 \mathrm{~min}$, and hybridization was conducted using a digoxin-labeled circRNF13 probe (Sangon, Biotech, Shanghai, China) overnight at $37^{\circ} \mathrm{C}$. The next day, slides were washed in $2 \times \mathrm{SSC}$, $0.5 \times \mathrm{SSC}$, and $0.2 \times \mathrm{SSC}$. Biotinylated rat anti-digoxigenin, streptavidin-biotin complex (sABC), and biotinylated peroxidase were then added dropwise. After incubation for $30 \mathrm{~min}$, slides were washed with PBS.
DAB color developing solution was subsequently added for 5-10 min and then placed in running water to stop the color reaction. Sections were subjected to dehydration using gradient alcohol, and a neutral resin mount was added dropwise. The $\operatorname{circRNF13}$ probe used is listed in Table S1.

\section{Protein half-life assay}

Initially, cells were transfected with the circRNF13 overexpression vector or circRNF13 siRNA for $48 \mathrm{~h}$. Then, cycloheximide (CHX, $50 \mu \mathrm{g} / \mathrm{mL}$ ) was added into cell culture medium at indicated time points.

\section{Immunoprecipitation}

For immunoprecipitation, the antibodies were incubated with $35 \mu \mathrm{L}$ of protein A/G magnetic beads (Bimake, Houston, Texas, USA) with constant rotation at room temperature for $2 \mathrm{~h}$. HNE2 or CNE2 cell lysates were extracted using $\mathrm{GLB}^{+}$lysis buffer $(150 \mathrm{mM} \mathrm{NaCl}, 10 \mathrm{mM}$ Tris- $\mathrm{HCl} \mathrm{pH}$ 7.5, 0.5\% Triton $\mathrm{X}-100,10 \mathrm{mM}$ EDTA pH 8.0) with a protease inhibitor cocktail (Roche, Basel, Switzerland, USA) on ice for $2 \mathrm{~h}$. Lysates were centrifuged and then incubated with antibody-conjugated beads for $4{ }^{\circ} \mathrm{C}$ overnight. Next the antibody-bead complexes were washed 5-6 times with cold $\mathrm{GLB}^{+}$lysis buffer. Then the precipitated proteins were resuspended and boiled $10 \mathrm{~min}$ using $6 \times$ SDSPAGE loading buffer. The boiled immune complex was put on ice for 2 min and subjected for SDS-PAGE electrophoresis. The primary antibodies used are listed in Table S2.

\section{Immunofluorescence}

The cultured HNE2 and CNE2 cells were incubated with $4 \%$ paraformaldehyde and then blocked with $5 \%$ BSA. The cells were treated with specific antibodies at $4{ }^{\circ} \mathrm{C}$ overnight and the secondary antibodies at $37^{\circ} \mathrm{C}$ for $1 \mathrm{~h}$. And the cells were counterstained with DAPI for $10 \mathrm{~min}$ and imaged under a confocal microscope (Ultra-View Vox, Perkin-Elmer, Waltham, MA, USA). The primary antibodies used are listed in Table S2.

\footnotetext{
(See figure on next page.)

Fig. 3 CircRNF13 inhibits NPC growth and metastasis in vivo. A Images of subcutaneous tumor formation in nude mice at 4 weeks. Mice were injected with $2 \times 10^{6} \mathrm{CNE} 2$ cells, with knockdown or overexpression of circRNF13. B, C Tumor volumes (B) and tumor weights (C) were measured for each group ( $n=7$ per group). Data have been represented as mean \pm SD. ${ }^{*}, p<0.05 ;{ }^{* *}, p<0.001$. D Representative images of in situ hybridization for circRNF13 and immunohistochemical staining for Ki-67 expression in subcutaneous tumors ( $200 \times$, scale bar: $50 \mu$ m). E Images of visible nodules on the lung surface. CNE2 cells transfected with scrambled siRNA, empty vector, sicircRNF13, or circRNF13 overexpression vector were injected into each nude mouse tail vein ( $n=7$ for each group), and the mice were sacrificed 8 weeks later. $\mathbf{F}$ The number of lung metastatic nodules on each lung surface was quantified. Data have been represented as mean \pm SD (each point represents one mouse; $n=7$ per group; right). ${ }^{* * *}, p<0.001$. G Representative images of circRNF13 expression, as assessed using in situ hybridization, and lung metastatic tumor foci after H\&E staining (200 x , scale bar: $50 \mu \mathrm{m})$
} 


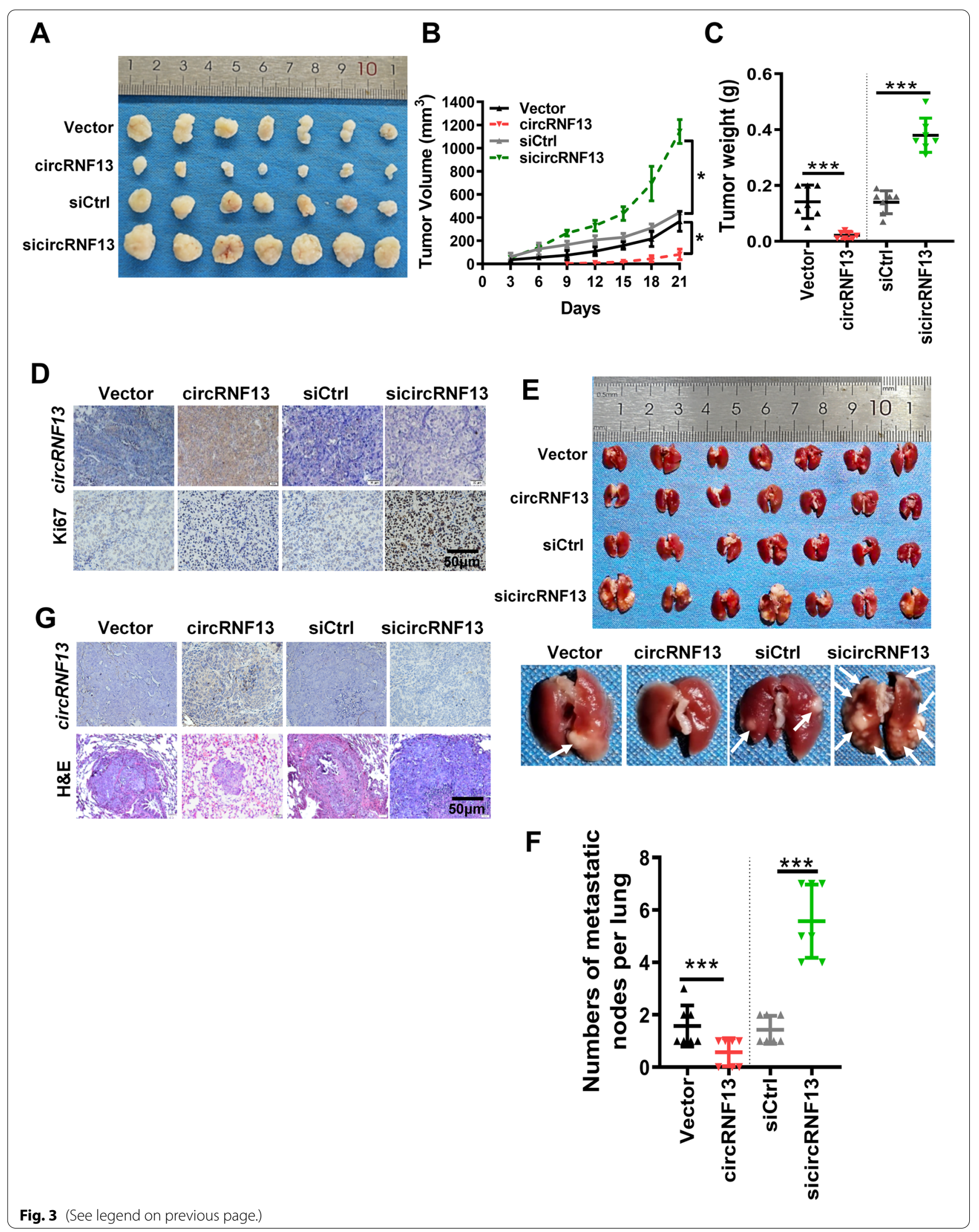




\section{Western blotting}

Total protein lysate (40 $\mu \mathrm{g}$ per lane) was loaded on 10-15\% Bis-tris polyacrylamide mini gels (Invitrogen). SDS-PAGE was run at $120 \mathrm{~V}$ for $1.5 \mathrm{~h}$ to $2 \mathrm{~h}$. Proteins were transferred to nitrocellulose or PVDF membranes by wet transfer for $60-90 \mathrm{~min}$ at $100 \mathrm{~V}$. Membranes were blocked in 5\% nonfat dry milk in Tris-buffered saline supplemented with Tween 20 (0.1\%) (TBST) or phosphate-buffered saline (PBS) for $60 \mathrm{~min}$ at room temperature. After blocking, membranes were cut horizontally to examine multiple proteins of different sizes on each gel. Membranes were incubated on a plate shaker overnight at $4{ }^{\circ} \mathrm{C}$ with primary antibodies diluted in TBS-T. Membranes were extensively washed with TBS-T (minimum $3 \times$ for $10 \mathrm{~min}$ ), followed by incubation with appropriate horseradish peroxidase-conjugated secondary antibodies diluted in TBS-T with $5 \%$ nonfat dry milk for $60 \mathrm{~min}$ at RT on a plate shaker. Membranes were extensively washed with TBS-T (minimum $4 \times$ for $15 \mathrm{~min}$ ). Signals were detected using a Luminata Crescendo detection system following the manufacturer's recommendations. Multiple film (HyBlot CL, Denville) exposures ranging from $2 \mathrm{~s}$ to 2 min were performed for optimal image analysis. The antibodies are listed in Table S2.

\section{Immunohistochemistry}

Immunohistochemistry was performed on formalin-fixed paraffin-embedded sections of mouse xenograft tissues. Briefly, tissues were deparaffinized and rehydrated, and samples were subjected to EDTA-mediated high-temperature antigen retrieval; the samples were then incubated overnight at $4{ }^{\circ} \mathrm{C}$ with the primary antibodies. The staining was scored according to the staining intensity and the distribution of stained cells. The distribution was evaluated as none (0), $\leq 10 \%(1), 10 \%-50 \%(2), 50 \%-80 \%$ (3), and $>80 \%$ (4). Intensity was evaluated as none (0), faint (1), moderate (2), strong (3), or very strong (4). The sections were reviewed by two pathologists. The antibodies are listed in Table S2.

\section{Statistical analysis}

Statistical analysis was performed using GraphPad Prism 7 software. Student's t-tests were used to evaluate significant differences between any two groups of data, and one-way ANOVA was used to evaluate significant differences for multiple comparisons. All data are represented as mean \pm Standard Deviation (SD). Differences were considered significant at $p<0.05$. ${ }^{*}, p<0.05$; ${ }^{* *}, p<0.01$; ***, $p<0.001$.

\section{Results}

\section{CircRNF13 exhibits low expression in NPC clinical tissues}

To obtain the expression profiles of circRNAs in NPC, next-generation sequencing of mRNA (RNAseq) data in the NPC cell line 5-8F (accession number: PRJNA391554) was reanalyzed [20]. A total of 6,153 distinct circRNAs were identified after excluding very low abundance circRNAs (average reads per million fragments mapped $<0.1$ ). Among these circRNAs, five types were included: exon-derived circRNAs, intronderived circRNAs, anti-sense strand circRNAs, overlapping regions of sense strand circRNAs, and intergenic circRNAs, according to their different looping regions (Fig. 1A). The expression of the top 20 circRNAs (Table $\mathrm{S} 3$ ), based on their abundance were assessed in $36 \mathrm{NPC}$ tissues and 12 normal nasopharyngeal mucosal/epithelial (NPE) tissues (Table S4), using RT-PCR. Among them, circMAN1A2, circFAM129B, circCAMSAP1, circ$C Y D L$, $\operatorname{circARARB1}$, circPVT1, and circSETD3 were highly expressed in the $36 \mathrm{NPC}$ tissues, as compared to the 12 NPE tissues (Fig. S1A). A novel potential circRNA, circRNF13, was expressed in high abundance in NPC tissues, but circRNF13 expression was higher in NPE tissues than in NPC tissues (Fig. 1B). Further experiments showed that circRNF13 was also expressed at lower levels in NPC cell lines (HNE2, CNE2, 5-8F, HONE1, and 6-10B) than in the normal NPE cell line NP69 (Fig. S1B). Based on the design of reverse primers for RT-PCR at the circRNF13 splice site and Sanger sequencing of the RT-PCR products, we concluded that

\footnotetext{
(See figure on next page.)

Fig. 4 CircRNF13 inhibits glycolysis in NPC cells. A Glucose consumption and lactate production in HNE2 and CNE2 cells were examined using an automated biochemical analyzer, upon circRNF13 overexpression. Data have been represented as mean \pm SD from three independent experiments. *, $p<0.05$. B The extracellular acidification rate (ECAR) in HNE2 and CNE2 cells was measured using Seahorse XF assay, in response to circRNF13 overexpression or knockdown. Glycolysis, glycolytic capacity, and glycolytic reserve were analyzed. Data have been represented as mean \pm SD. ${ }^{*}, p<0.05 ;{ }^{* *}, p<0.01$. C Expression levels of downstream targets of phosphorylated AMPKa and mTOR, S6K and 4EBP1, were measured using western blotting in HNE2 and CNE2 cells in response to circRNF13 overexpression or knockdown. D Expression levels of downstream targets of phosphorylated AMPKa and mTOR, S6K and 4EBP1, were measured using western blotting in HNE2 and CNE2 cells treated with the glycolysis inhibitor 2-DG, in response to circRNF13 overexpression or knockdown. E The glycolysis inhibitor 2-DG attenuated the effect of circRNF13 on NPC cell migration in HNE2 and CNE2 cells transfected with sicircRNF13, as assessed using wound-healing assay. All experiments were repeated at least three times. Data have been represented as mean \pm SD. ${ }^{* * *}, p<0.001$. F The glycolysis inhibitor 2-DG weakened the function of circRNF13 on NPC cell invasion when HNE2 and CNE2 cells transfected with sicircRNF13 were treated with the glycolysis inhibitor 2-DG, as assessed using Transwell assays. All experiments were repeated at least three times. Data have been represented as mean \pm SD. ${ }^{* * *}, p<0.001$
} 

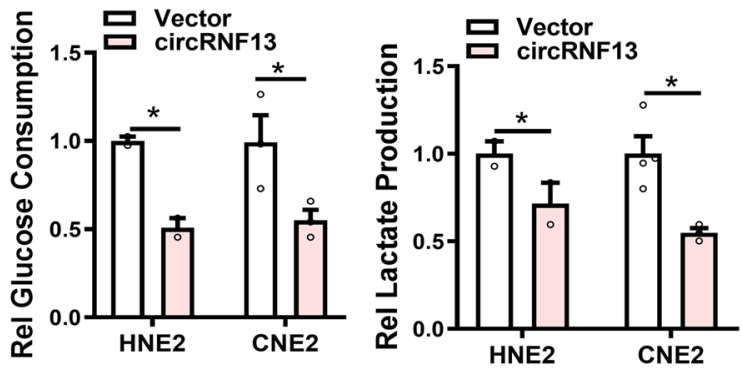

C

A

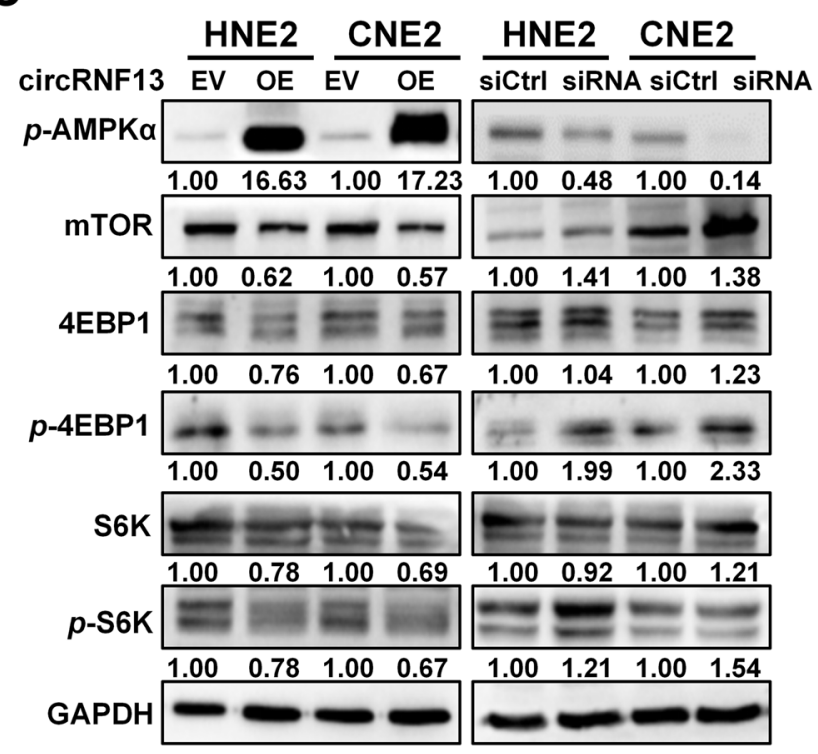

D

HNE2

circRNF13 Ctrl siRNA Ctrl siRNA Ctrl siRNA Ctrl siRNA

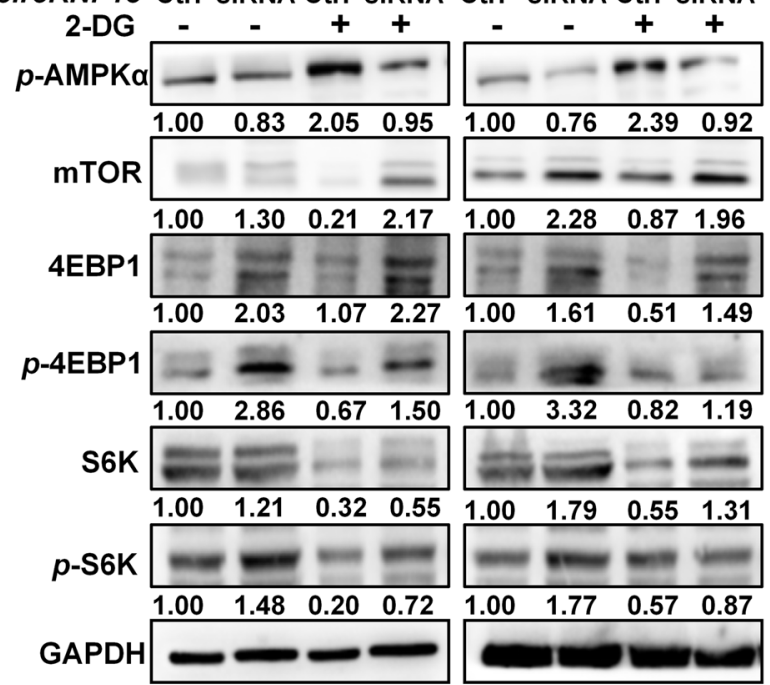

B

HNE2

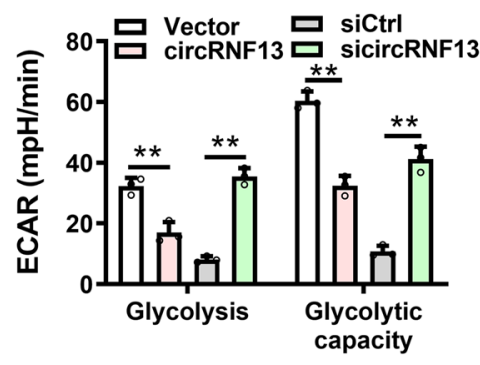

CNE2

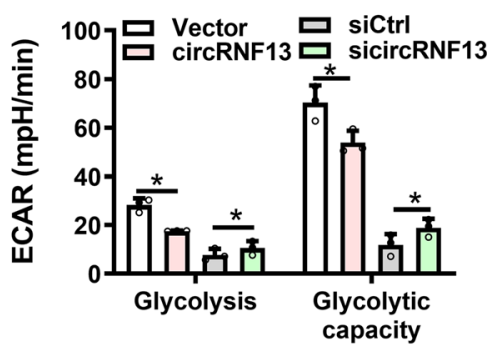

Migration
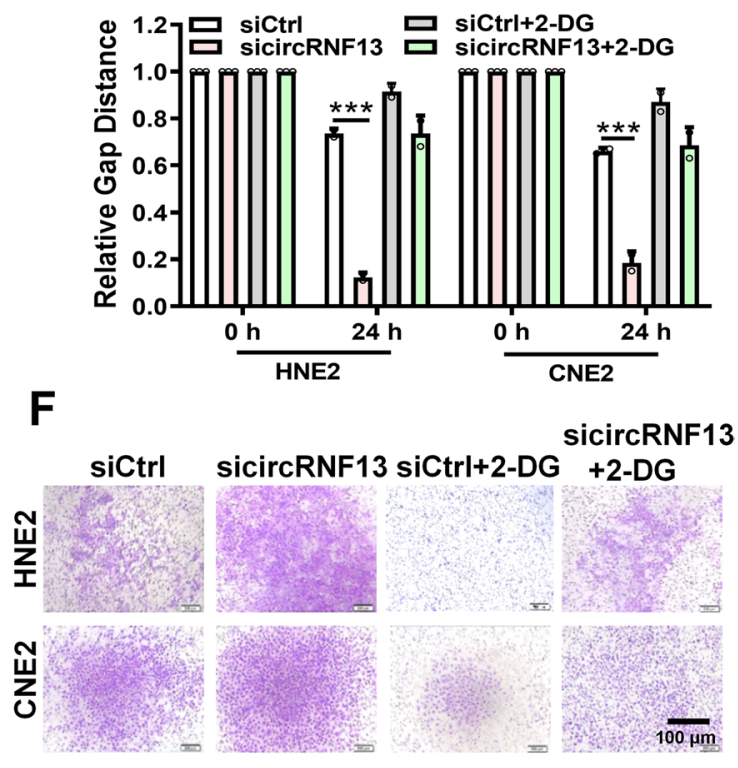

Invasion

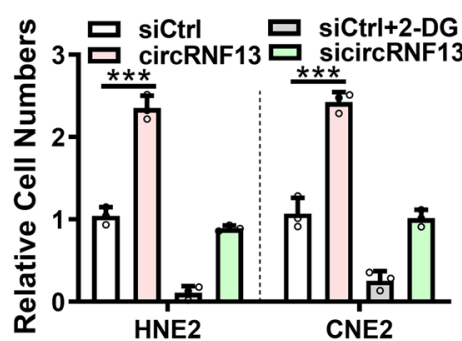

Fig. 4 (See legend on previous page.) 
circRNF13 is formed by circular splicing of the exons 2-8 of RNF13 gene (NM_183381.2) on the chromosome $3 q 25.1$, with a full length of 716 bp (Fig. 1C). Nucleoplasmic separation assays and RNA fluorescence in situ hybridization assays showed that circRNF13 was distributed in both the nucleus and cytoplasm and was more localized in the nucleus. (Fig. 1D-E), while RNase R treatment revealed that circRNF13 was more stable than RNF13 mRNA (Fig. 1F) in NPC cells. These data suggested that circRNF13 may affect NPC development.

\section{CircRNF13 inhibits proliferation, migration, and invasion of NPC cells}

To explore the function of $\operatorname{circRNF13}$ in NPC, a circRNF13 overexpression vector was constructed, and a circRNF13 siRNA was designed based on the splice site of $\operatorname{circRNF13.~RT-PCR~results~showed~that~} \operatorname{circRNF13}$ overexpression vector or circRNF13 siRNA successfully overexpressed or knocked down circRNF13 in NPC cells and had no effect on linear RNF13 mRNA (Fig. S2). MTT and colony formation assays showed that overexpression of circRNF13 inhibited NPC cell proliferation, and knockdown of circRNF13 promoted NPC cell proliferation (Fig. 2A, B). Flow cytometry results also showed that cells were stalled in the $\mathrm{G} 1$ phase upon overexpression of circRNF13, while knockdown of circRNF13 increased the number of cells in the G2/M phase (Fig. 2C). Results of the wound-healing experiments showed that the migration capacity of NPC cells was significantly reduced in response to high circRNF13 expression and induced in response to low circRNF13 expression (Fig. 2D). Transwell assays showed that overexpression of $\operatorname{circRNF13}$ inhibited NPC cell invasion and knockdown of circRNF13 promoted invasion (Fig. 2E). Based on these observations, we concluded that circRNF13 inhibits NPC cell proliferation, migration, and invasion.

\section{CircRNF13 inhibits NPC growth and metastasis in vivo}

Next, we investigated the effect of circRNF13 on NPC cell proliferation and metastasis in vivo. CNE2 cells transfected with sicircRNF13 or circRNF13 overexpression vector were inoculated into female nude mice, to establish a xenograft model, by means of subcutaneous or tail vein injection, respectively. The results of subcutaneous tumorigenesis experiments showed that the circRNF13-overexpressing nude mice exhibited smaller tumors, while the circRNF13 knockdown group presented larger subcutaneous tumors than the control group (Fig. 3A-C). Paraffin embedding, sectioning, and immunohistochemical staining showed that Ki-67 expression in the circRNF13 overexpression group was significantly lower than that in the circRNF13 knockdown group (Fig. 3D). The results of the tail vein lung metastasis assay showed that the number of lung nodules in the circRNF13 overexpression group was significantly lower than that in the control group, while there was an increase in the number of lung nodules in the circRNF13 knockdown group (Fig. 3E-F). H\&E staining of lung nodules also revealed that the number of NPC lung metastases in the circRNF13 overexpression group was significantly lower than that in the circRNF13 knockdown group (Fig. 3G). Taken together, the above results suggested that circRNF13 inhibits the proliferation and metastasis of NPC in vitro and in vivo.

\section{CircRNF13 may inhibit migration and invasion of NPC by inhibiting glycolysis}

To investigate the molecular mechanism of $\operatorname{circRNF13}$ on NPC proliferation and metastasis, we performed liquid chromatography-tandem mass spectrometry (LCMS/MS) in CNE2 cells transfected with sicircRNF13 or scrambled siRNA. The score and area values of the LC-MS/MS data were selected as the evaluation criteria to compare proteins' expression between the two groups. A total of 294 differentially expressed proteins were identified (Table S5). Enrichment analysis by IPA (Ingenuity Pathway Analysis) software showed that many molecules in the metabolic-related pathways, especially the glycolysis pathway, were included (Fig. S3A-B). These results imply that the glycolysis pathway might be regulated by $\operatorname{circRNF13}$ in NPC.

To explore the function of $\operatorname{circRNF13}$ in the glycolysis pathway, biochemical assays were performed, which revealed that overexpression of circRNF13 inhibited glucose uptake and lactate production in NPC cells (Fig. 4A). Seahorse assay revealed that overexpression of circRNF13 caused a significant decrease in the glycolytic capacity of CNE2 and HNE2 cells, while knockdown of circRNF13 significantly increased glycolysis (Fig. 4B, Fig. S3C).

\section{(See figure on next page.)}

Fig. 5 CircRNF13 inhibits glycolysis in NPC cells by promoting GLUT1 ubiquitination. A The mRNA levels of GLUT1, HK2, and LDHA in HNE2 and CNE2 cells were examined using RT-PCR, upon overexpression and knockdown of circRNF13. Data have been represented as mean \pm SD; ns, no significance; ***, $p<0.001$. B The expression levels of GLUT1, HK2, and LDHA proteins were examined using western blotting in HNE2 and CNE2 cells, upon overexpression and knockdown of circRNF13. C Degradation of GLUT1 was detected in HNE2 and CNE2 cells using western blotting, after overexpression and knockdown of circRNF13 and treatment with $50 \mu \mathrm{g} / \mathrm{mL}$ cycloheximide (CHX). D Ubiquitination levels of GLUT1 protein were detected in HNE2 and CNE2 cells using pull-down with an anti-Flag antibody and western blot with an anti-ubiquitin antibody, after overexpression or knockdown of circRNF13 


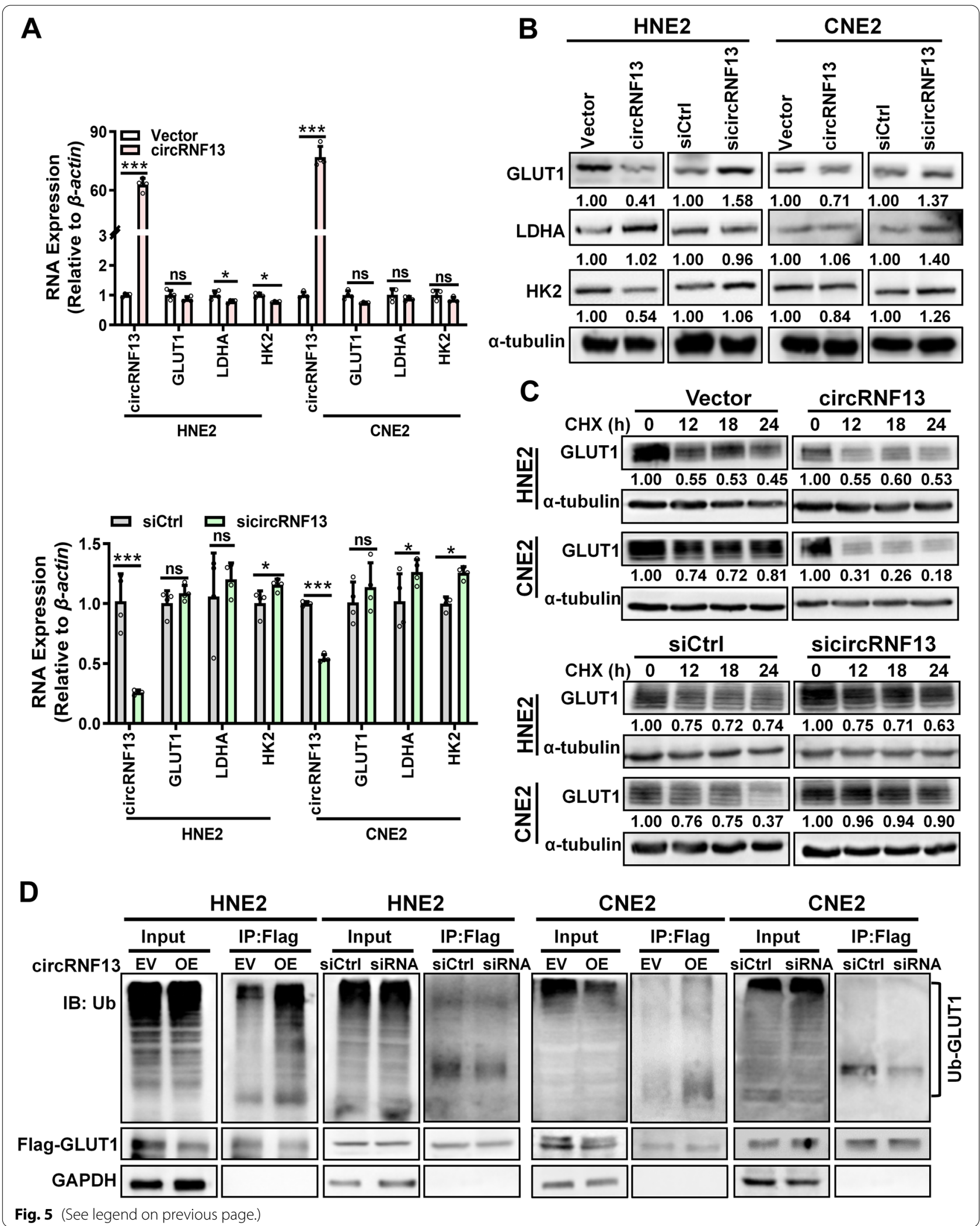


The glycolysis pathway can be monitored by measuring the intracellular AMPK levels. When the glycolysis process is inhibited, intracellular AMPK levels are activated by phosphorylation, and activated AMPK inhibits protein synthesis and translation by inhibiting mTOR, which in turn inhibits tumor growth and metastasis $[16,17]$. To further clarify whether cricRNF13 inhibits the proliferation and migration of NPC cells by inhibiting glycolysis, the intracellular phosphorylation of AMPK and mTOR was examined in HNE2 and CNE2 cells. Western blotting revealed that overexpression of circRNF13 in NPC cells significantly upregulated phosphorylated AMPK $\alpha$ and downregulated mTOR, further downregulating the expression of downstream targets of mTOR, pS6K and 4EBP1. Knockdown of circRNF13, on the other hand, induced the opposite effect (Fig. 4C). The glycolysis inhibitor 2-DG was used to block the glycolysis pathway. When the glycolysis inhibitor 2-DG was added, knockdown of circRNF13 did not downregulate phosphorylated AMPK $\alpha$ (Fig. 4D). This data revealed that knockdown of $\operatorname{circRNF13}$ promoted NPC cell migration and invasion, and this effect was diminished upon addition of 2-DG (Fig. 4E-F, Fig. S3D). These results suggested that circRNF13 may inhibit the migration and invasion of NPC by inhibiting glycolysis.

\section{CircRNF13 inhibits glycolysis in NPC cells by promoting GLUT1 ubiquitination}

To investigate the molecular mechanisms by which circRNF13 regulates the glycolytic process in NPC cells, the primary molecules of the glycolysis pathway, including GLUT1, LDHA, and HK2, were examined using RT-PCR and western blotting experiments in HNE2 and CNE2 cells. The data revealed that GLUT1 protein levels were significantly reduced in response to overexpression of circRNF13 and significantly increased upon knockdown of circRNF13, while the mRNA expression levels were not significantly altered. CircRNF13 had a weak effect on the expression of LDHA and HK2, at both the mRNA and protein levels (Fig. 5A, B).These data imply that circRNF13 may regulate glycolysis primarily through the modulation of GLUT1.
We speculated whether circRNF13 affected glycolysis by influencing the protein stability of GLUT1, so CNE2 and HNE2 cells were treated with cycloheximide $(\mathrm{CHX})$ after overexpression or knockdown of circRNF13. Western blotting showed that the stability of GLUT1 decreased in response to overexpression of circRNF13, while it increased upon knockdown of circRNF13 (Fig. 5C). Overexpression of circRNF13 promoted GLUT1 ubiquitination, whereas knockdown of circRNF13 decreased protein ubiquitination levels of GLUT1, as demonstrated using an anti-ubiquitin antibody (Fig. 5D). These results suggested that circRNF13 may inhibit glycolysis in NPC cells by regulating ubiquitin-mediated degradation of GLUT1.

\section{CircRNF13 promotes the stability of SUMO2 by directly binding to the $3^{\prime}$-UTR of SUMO2 mRNA}

To investigate how circRNF13 regulates GLUT1 ubiquitination, the LC-MS/MS data were reanalyzed, upon which SUMO2 caught our attention (Table S6). SUMO2 is a member of the SUMO family. SUMOylation is a type of ubiquitin-like protein modification that regulates protein degradation via the ubiquitination pathway. To explore the function of SUMO2 in NPC cells, SUMO2 was overexpressed in NPC cells; the overexpression was confirmed using RT-PCR and western blotting (Fig. S4A). The MTT assay showed that SUMO2 promoted NPC cell proliferation (Fig. S4B). Wound-healing and Transwell assays also demonstrated that SUMO2 induced NPC cell migration and invasion (Fig. S4C-D). Seahorse assays showed that SUMO2 inhibited glycolysis in NPC (Fig. S4E). Therefore, these data indicated that SUMO2 inhibits glycolysis in NPC.

To identify whether $\operatorname{circRNF13}$ interacts with SUMO2 to regulate proliferation, migration, and invasion, western blotting and RT-PCR were performed, which revealed that overexpression of circRNF13 significantly induced the expression of SUMO2 at both the RNA and protein levels. The opposite results were obtained upon knockdown of circRNF13 (Fig. 6A, B). Bioinformatics analysis revealed the presence of a continuous binding motif between $\operatorname{circRNF13}$ and the 3'-UTR of SUMO2 (Fig. 6C).

\footnotetext{
(See figure on next page.)

Fig. 6 CircRNF13 directly binds to and stabilizes SUMO2 mRNA, to upregulate its expression A. Expression of SUMO2 protein was examined using western blotting in HNE2 and CNE2 cells, after overexpression and knockdown of circRNF13. B Expression of SUMO2 mRNA was examined in HNE2 and CNE2 cells using RT-PCR, after overexpression and knockdown of circRNF13. C Bioinformatics prediction using the RNAhybrid website revealed that circRNF13 has a continuous binding site with the 3'-UTR of SUMO2 mRNA. D circRNF13 was found to bind to the 3'-UTR of SUMO2 mRNA in HNE2 and CNE2 cells using RNA pull-down assay. GADPH was used as a negative control. Data have been represented as mean \pm SD. ${ }^{* * *}, p<0.001$. ECircRNF13 enhanced the luciferase activity of the SUMO2 mRNA 3'-UTR in HNE2 and CNE2 cells, as assessed using dual luciferase reporter assay. The 3'-UTR of GADPH was used as a negative control. Data have been represented as mean \pm SD. ${ }^{*}, p<0.05 ;{ }^{* *}, p<0.01$. F. Degradation of SUMO2 was detected in HNE2 and CNE2 cells using RT-PCR, after overexpression and knockdown of circRNF13 and treatment with $2 \mu \mathrm{g} / \mathrm{mL}$ actinomycin D. Data have been represented as mean \pm SD. ${ }^{* *}, p<0.001,{ }^{* * *}, p<0.0001$
} 
A

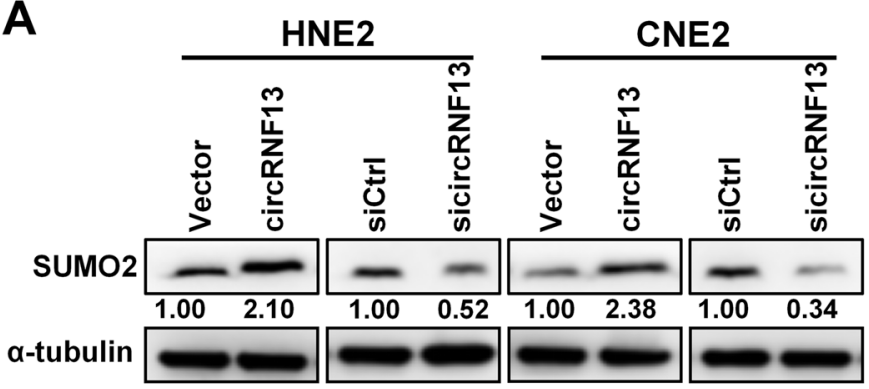

C

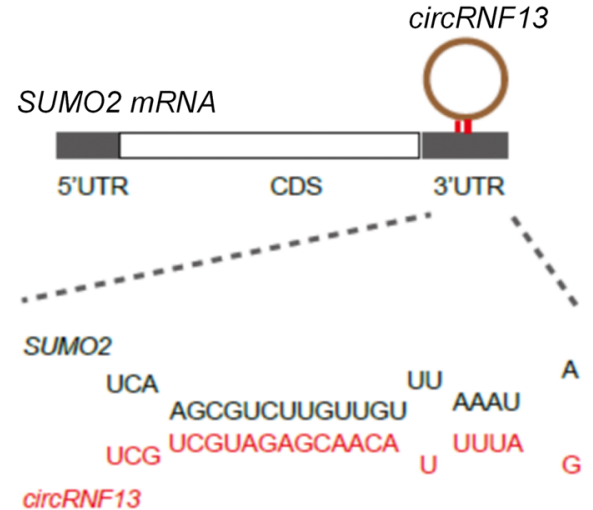

D

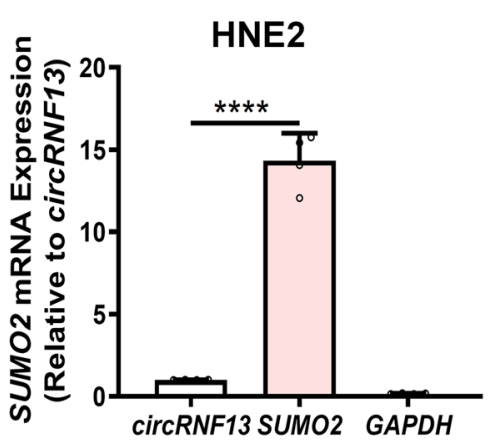

E

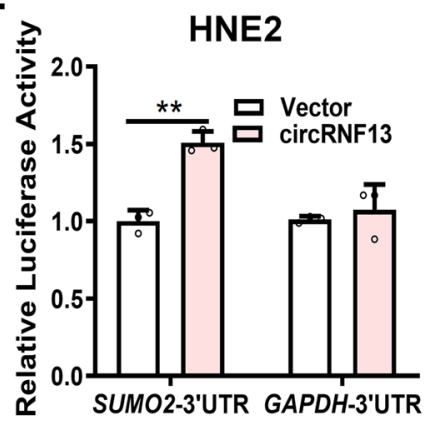

$\mathbf{B}$
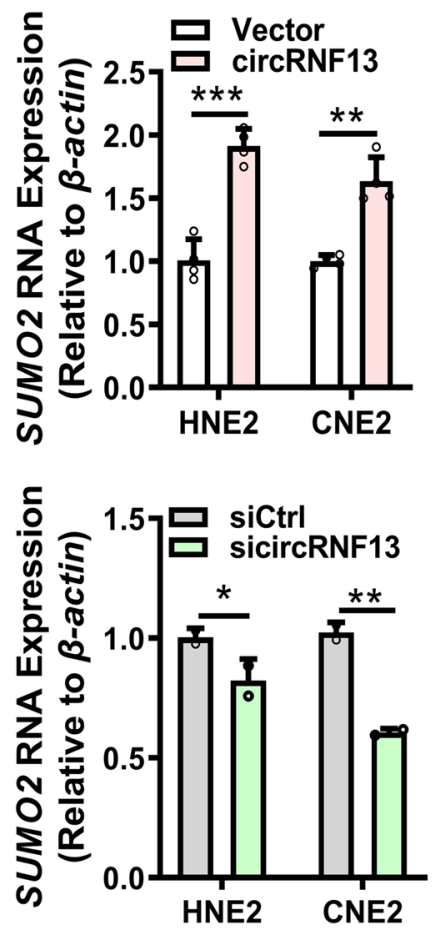

F

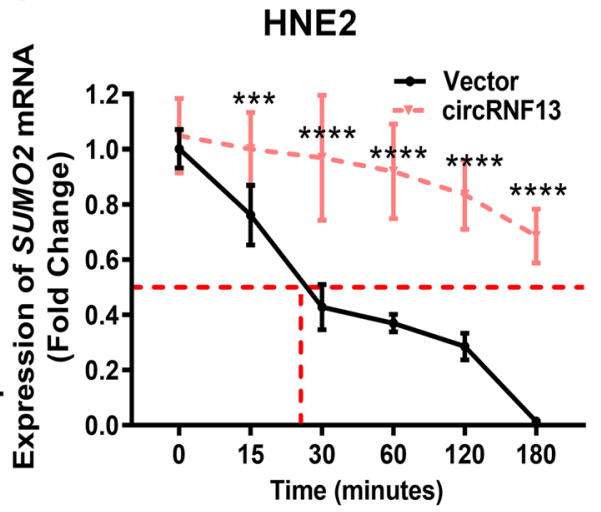

CNE2
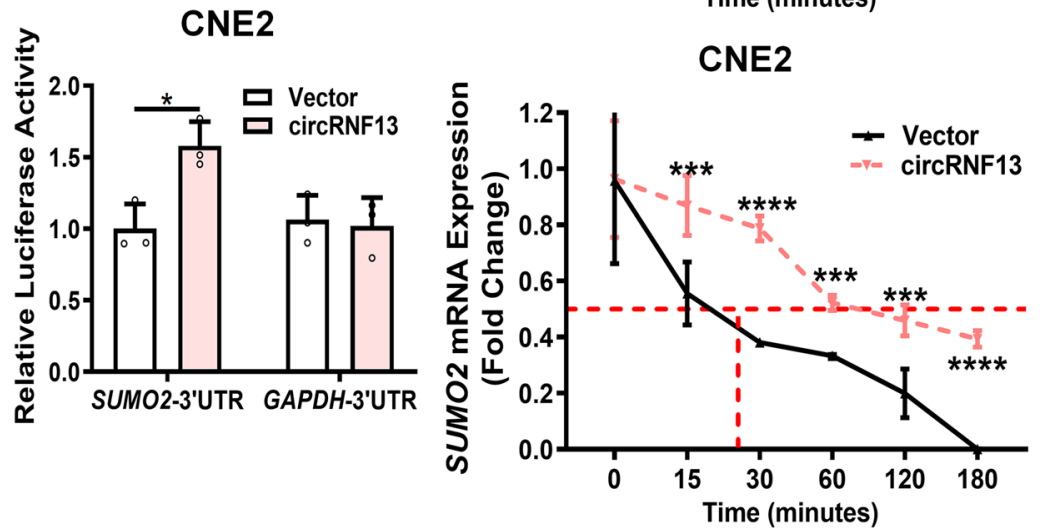

Fig. 6 (See legend on previous page.) 
RNA pull-down assays demonstrated that biotin-labeled circRNF13 could pull-down SUMO2 mRNA in NPC cells (Fig. 6D). Dual-luciferase experiments demonstrated that overexpression of circRNF13 increased the fluorescence activity of the SUMO2 3'-UTR in CNE2 cells (Fig. 6E). Actinomycin D $(2 \mu \mathrm{g} / \mathrm{mL})$ was used to measure whether circRNF13 regulates the stability of SUMO2 by binding to its $3^{\prime}$-UTR in HNE2 and CNE2 cells, upon overexpression of circRNF13. Compared to the control group, the stability of SUMO2 mRNA was significantly increased in the circRNF13 overexpression group (Fig. 6F). These data indicated that circRNF13 regulates SUMO2 expression by binding to its $3^{\prime}$-UTR.

\section{CircRNF13 promotes ubiquitination of GLUT1 via SUMO2}

To investigate whether circRNF13 regulates GLUT1 ubiquitination by binding to SUMO2, bioinformatics analysis was performed and revealed that there were two binding motifs of SUMO2 protein on the GLUT1 protein (Fig. S5A). Co-immunoprecipitation (Co-IP) experiments were performed to confirm the interaction between GLUT1 and the SUMO2 protein using an anti-FlagGLUT1 antibody. During SUMOylation, UBC9 is the only ligase that can directly recognize the substrate and catalyze the binding of the $\mathrm{C}$-terminal carboxyl group of the SUMO protein to the substrate protein for modification. Our Co-IP experiment also revealed an interaction between GLUT1 and UBC9 (Fig. 7A,B). Immunofluorescence also revealed co-localization of the SUMO2 and GLUT1 proteins (Fig. 7C). To understand the molecular basis of SUMO2 in NPC, SUMOylation of GLUT1 was examined in HNE2 and CNE2 cells, upon overexpression or knockdown of circRNF13. Western blotting showed that overexpression of circRNF13 promoted SUMOylation of GLUT1, and knockdown of circRNF13 inhibited SUMOylation of GLUT1 (Fig. 7D).

To determine whether SUMO2 participates in the

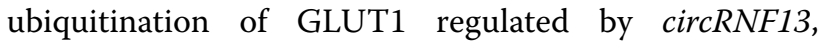
SUMO2 was overexpressed in NPC cells; western blotting showed that overexpression of SUMO2 inhibited
GLUT1 expression (Fig. S5B). Overexpression of SUMO2 induced GLUT1 degradation in HNE2 and CNE2 cells treated with cycloheximide (CHX) (Fig. S5C). Ubiquitination experiments demonstrated that overexpression of SUMO2 accelerated the ubiquitination of GLUT1 (Fig. 7E). Knockdown of circRNF13 weakened the SUMO2-induced ubiquitination level of GLUT1 in HNE2 and CNE2 cells co-transfected with sicircRNF13 and SUMO2 overexpression vector (Fig. 7F). These results suggested that $\operatorname{circRNF13}$ promotes the ubiquitination of GLUT1, to inhibit GLUT1 expression, thus suppressing the glycolytic process in NPC cells. These data suggested that circRNF13 regulates GLUT1 ubiquitination by binding to SUMO2.

\section{CircRNF13 inhibits proliferation, migration, and invasion of NPC cells via SUMO2}

MTT assays were performed to determine whether circRNF13 inhibits NPC cell proliferation, migration, and invasion via SUMO2, which revealed that overexpression of SUMO2 rescued the circRNF13 knockdownmediated altered proliferation ability of NPC cells (Fig. 8A). Wound-healing and Transwell assays showed that SUMO2 rescued the circRNF13 knockdown-mediated altered migration and invasive abilities of NPC cells (Fig. 8B, C). Seahorse assay also showed that overexpression of SUMO2 rescued the altered glycolytic stress in NPC cells caused by knockdown of circRNF13 (Fig. 8D).

Immunohistochemistry for SUMO2 and GLUT1 was also performed in mouse tissues, which showed that SUMO2 was expressed at low levels, while GLUT1 was highly expressed in subcutaneous tumor sections of nude mice in the circRNF13 overexpression group (Fig. 8E). These results underscored the significance of $\operatorname{circRNF13}$ in NPC proliferation and metastasis. circRNF13, acting as a tumor suppressor, directly binds to the $3^{\prime}$-UTR of SUMO2 and prolongs the half-life of SUMO2 mRNA. Upregulation of SUMO2 promotes GLUT1 degradation through SUMOylation and ubiquitination of GLUT1, which regulates the AMPK-mTOR pathway by inhibiting

\footnotetext{
(See figure on next page.)

Fig. 7 CircRNF13 promotes ubiquitination of GLUT1 via SUMO2. A The interaction between GLUT1, SUMO2, and UBC9 was examined using immunoprecipitation and an anti-Flag (GLUT1) antibody in HNE2 and CNE2 cells transfected with Flag-GLUT1 vector, followed by western blotting using the anti-SUMO2 and anti-UBC9 antibodies. B The interaction between SUMO2, GLUT1, and UBC9 proteins was examined using immunoprecipitation with an anti-HA (SUMO2) antibody in HNE2 and CNE2 cells transfected with HA-SUMO2 vector, followed by western blotting using anti-GLUT1 and anti-UBC9 antibodies. C Immunofluorescence experiments performed using anti-Flag (GLUT1) and anti-SUMO2 antibodies in HNE2 and CNE2 cells showed that GLUT1 and SUMO2 were co-localized. DAPI-stained nucleus: blue; anti-Flag (GLUT1): red; anti-SUMO2: green; co-localization of GLUT1 and SUMO2: yellow; the merged image represents the overlap of DAPI, GLUT1, and SUMO2 (scale bar: $20 \mu \mathrm{m}$ ). D Immunoprecipitation experiments using an anti-Flag-GLUT1 antibody, followed by western blotting using an anti-SUMO2 antibody, were performed, to identify the SUMOylation level of the GLUT1 protein in HNE2 and CNE2 cells transfected with sicircRNF13 or circRNF13 overexpression vector. E The ubiquitination level of GLUT1 protein was detected in HNE2 and CNE2 cells using pull-down with an anti-Flag-GLUT1 antibody, followed by western blotting with an anti-ubiquitin antibody, to determine whether SUMO2 affects GLUT1 ubiquitination. F A pull-down experiment using an anti-Flag-GLUT1 antibody, followed by western blot using an anti-ubiquitin antibody, were performed to identify the ubiquitination level of GLUT1 protein in HNE2 and CNE2 cells co-transfected with sicircRNF13 and SUMO2 overexpression vector
} 
A

HNE2

CNE2

Input IgG IP:Flag Input IgG IP:Flag

Flag-GLUT1

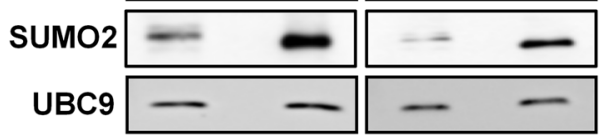

B

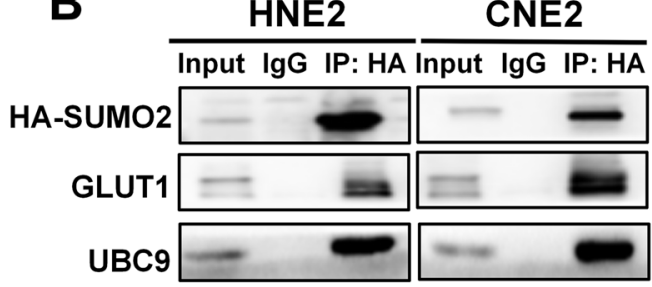

C
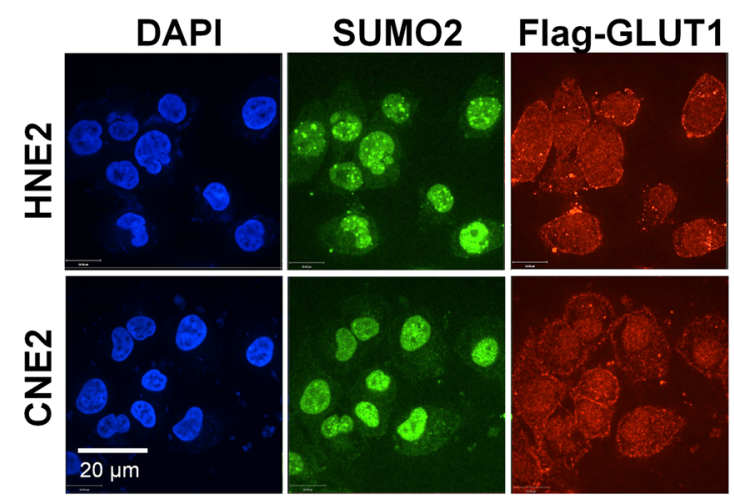

Merge

Colocalize
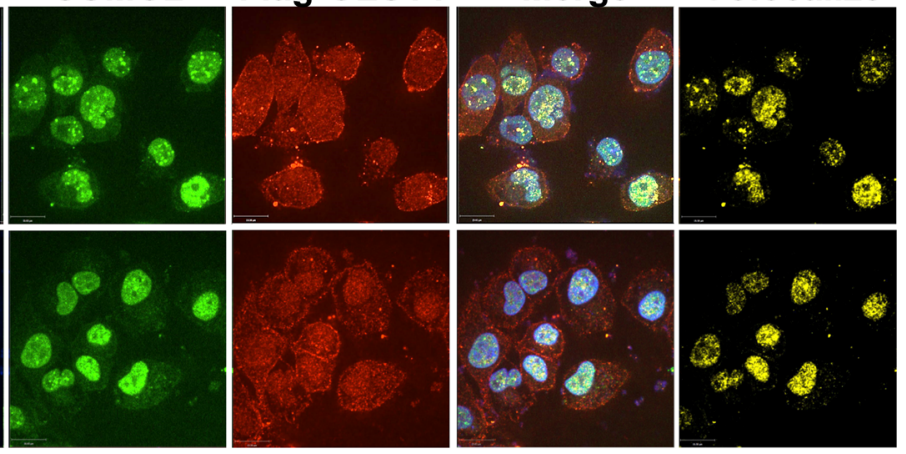

D

HNE2

\section{CNE2}

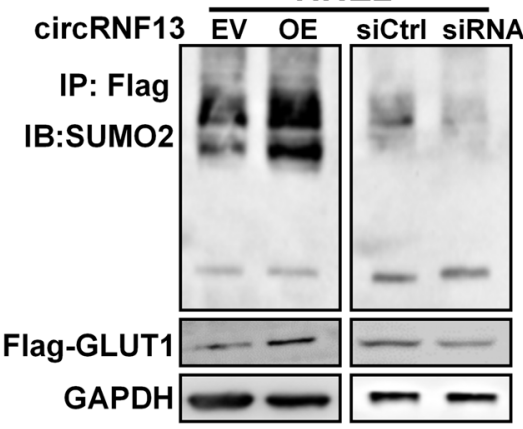

E

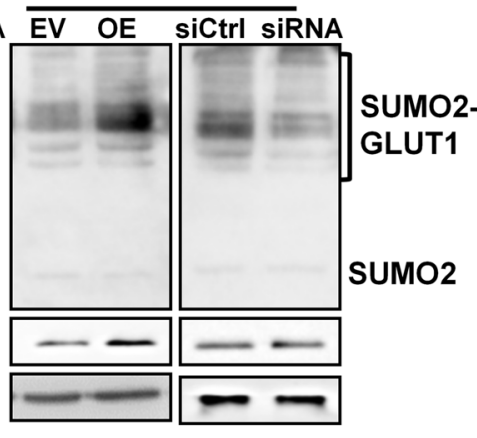

E

HNE2

CNE2

F $\overline{\frac{\text { Input }}{-+} \frac{\text { IP:Flag }}{-+}} \frac{\text { Input }}{-+\frac{\text { IP:Flag }}{-+t}}$

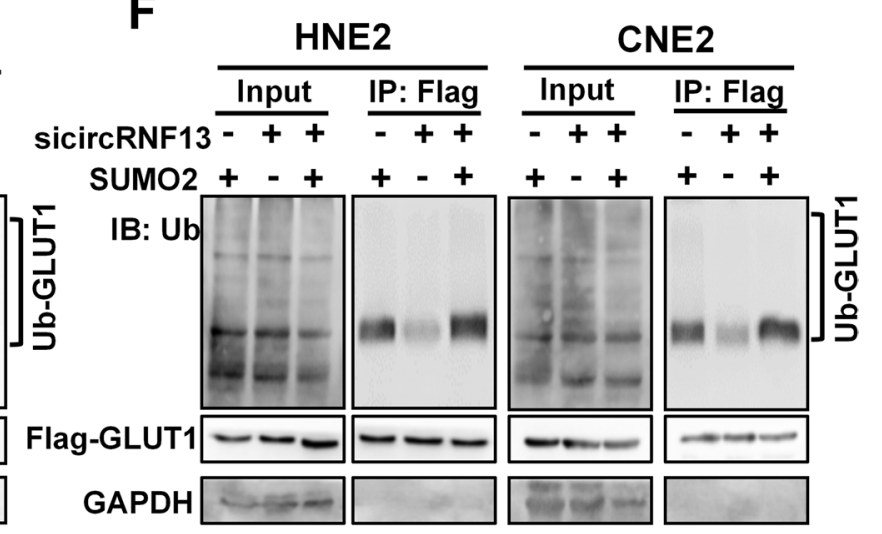

Flag-GLUT1

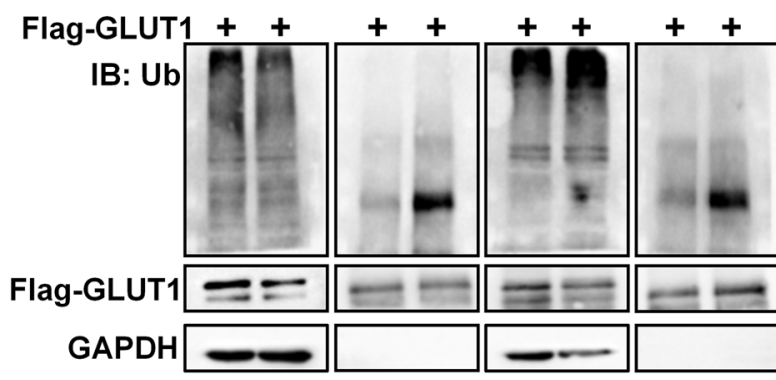
$++$ $+\quad+\quad+$

Fig. 7 (See legend on previous page.) 
glycolysis, ultimately resulting in proliferation and metastasis of NPC (Fig. 8F).

\section{Discussion}

In this study, we identified a new circRNA, circRNF13, which is expressed at low levels in NPC and regulates glycolysis in NPC cells by binding to the 3'-UTR of SUMO2 mRNA, to enhance GLUT1 degradation, resulting in the proliferation and metastasis of NPC. We screened the circRNAs from our RNA-seq data on the NPC cell line 5-8F. From the RNA-seq data, some new circRNAs were identified, which have not been reported yet. Some of the other identified circRNAs have already been reported to play important functions in the development of NPC. For example, circMAN1A2 is highly expressed in the serum of patients, including NPC patients, and can be used as a potential molecular marker for tumors [24].

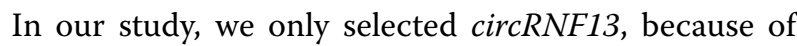
its high abundance in NPC cells. However, it was more highly expressed in NPE tissues than in NPC tissues. In NPC, some circRNAs have been reported to be oncogenes that promote the development of NPC. Our previous data showed that circSETD3, acting as a competing endogenous RNA, promotes NPC migration and invasion by completely binding to $m i R-615-5 p$ and $m i R-1538$ with MAPRE1 [25]. CircCRIM1 promotes NPC metastasis and chemoresistance by upregulating FOXQ1 expression through adsorption of $m i R-422 a$ [26]. Wang et al. found that circTGFBR2 acted as a sponge for $m i R-107$, to upregulate TGFBR2, resulting in the inhibition of NPC progression [27]. Chen et al. found that circHIPK3 promoted NPC proliferation and invasion by eliminating $m i R-4288$-induced inhibition of ELF3 [28]. Fan et al. found that circARHGAP12 promoted NPC cell invasion and metastasis by binding directly to the $3^{\prime}$-UTR of EZR mRNA and promoting its stability [29]. These results suggested that circRNAs play an important role in promoting the development of NPC. In this study, we found that circRNF13, acting as a tumor suppressor, directly binds to and stabilizes SUMO2 mRNA, to promote SUMO2 protein expression, thereby inhibiting the proliferation and metastasis of NPC. Thus, it can serve as a new model for research on the mechanism of circRNA regulation in tumor development and for the development of drug targets.

Metabolic reprogramming and invasive metastasis are important features of tumors that have long been the focus of attention. Recent studies have revealed that these two malignant phenotypes are not independent, but are actually closely linked. For example, the transmembrane glycoprotein CD147 promotes MCT1 expression, leading to increased lactate secretion, which activates the PI3K/ Akt/MDM2 pathway, increasing p53 degradation and promoting the proliferation and metastasis of hepatocellular carcinoma cells [30, 31]. PGC1- $\alpha$, a core regulator of metabolism, is significantly overexpressed in cancer and leads to a significant increase in tumor invasion and metastasis [32]. Morrison et al. found that melanomas with high expression of the monocarboxylate transporter MCT1 could resist oxidative stress by taking up lactic acid in the circulatory system, resulting in a greater metastatic capacity [33]. In conclusion, abnormal metabolic function of tumor cells can enhance tumor invasion and metastasis through multiple pathways. In this study, we found that circRNF13 inhibited glycolysis in NPC cells by suppressing the expression of GLUT1, which inhibited proliferation and metastasis. GLUT1 is an important protein in the process of glycolysis, and malignant tumor cells characteristically overexpress GLUT1, which provides favorable conditions for glycolysis through massive glucose uptake, thus providing more energy and synthetic raw materials for tumor cells. In addition, the large amount of lactic acid produced by glycolysis alters the microenvironment of tumor cells, which is more favorable for tumor cell invasion and metastasis. Designing circRNF13-based inhibitors against GLUT1, to block tumor cell metabolism, is also a promising strategy for future malignancy treatment.

\footnotetext{
(See figure on next page.)

Fig. 8 CircRNF13 inhibits the proliferation and metastasis of NPC via SUMO2. A Overexpression of SUMO2 rescued the circRNF13 knockdown-mediated altered proliferative capacity of HNE2 and CNE2 cells, as assessed using MTT assay. Data have been represented as mean \pm SD. ${ }^{* *}, p<0.01 ;{ }^{* *}, p<0.001$. B Overexpression of SUMO2 decreased the circRNF13 knockdown-mediated altered migration of HNE2 and CNE2 cells, as assessed using wound-healing assays. All experiments were repeated at least three times (Scale bar: $100 \mu \mathrm{m}$ ). Data have been represented as mean \pm SD; $n s$, no significance; ${ }^{* * *}, p<0.001$. C. Overexpression of SUMO2 reduced the circRNF13 knockdown-mediated altered invasive ability of HNE2 and CNE2 cells, as assessed using Transwell assays. All experiments were repeated at least three times (Scale bar: $100 \mu \mathrm{m}$ ). Data have been represented as mean $\pm S D ; n s$, no significance; ***, $p<0.001$. D The extracellular acidification rate (ECAR) was measured using Seahorse XF assay in HNE2 and CNE2 cells co-transfected with sicircRNF13 and SUMO2 overexpression vector. Glycolysis, glycolytic capacity, and glycolytic reserve were analyzed. All experiments were repeated at least three times. Data have been represented as mean $\pm \mathrm{SD} ; \mathrm{ns}$, not significant; ${ }^{*}, p<0.01$. E Representative images of SUMO2 and GLUT1 expression in lung tissues of nude mice, as determined using immunohistochemistry ( $n=7$ per group, $200 \times$, scale bar: $50 \mu \mathrm{m}$ ). F Schematic diagram of the molecular mechanism of circRNF13 in NPC. circRNF13 may activate and stabilize the SUMO2 protein by binding to the 3'-UTR of SUMO2 mRNA. Upregulation of SUMO2 promotes GLUT1 degradation through SUMOylation and ubiquitination of GLUT1, which regulates the AMPK pathway by inhibiting glycolysis, ultimately resulting in the proliferation and metastasis of NPC
} 


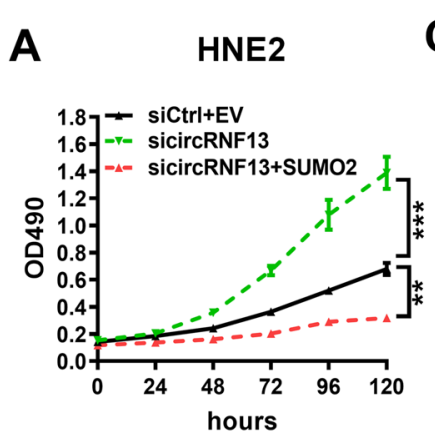

CNE2
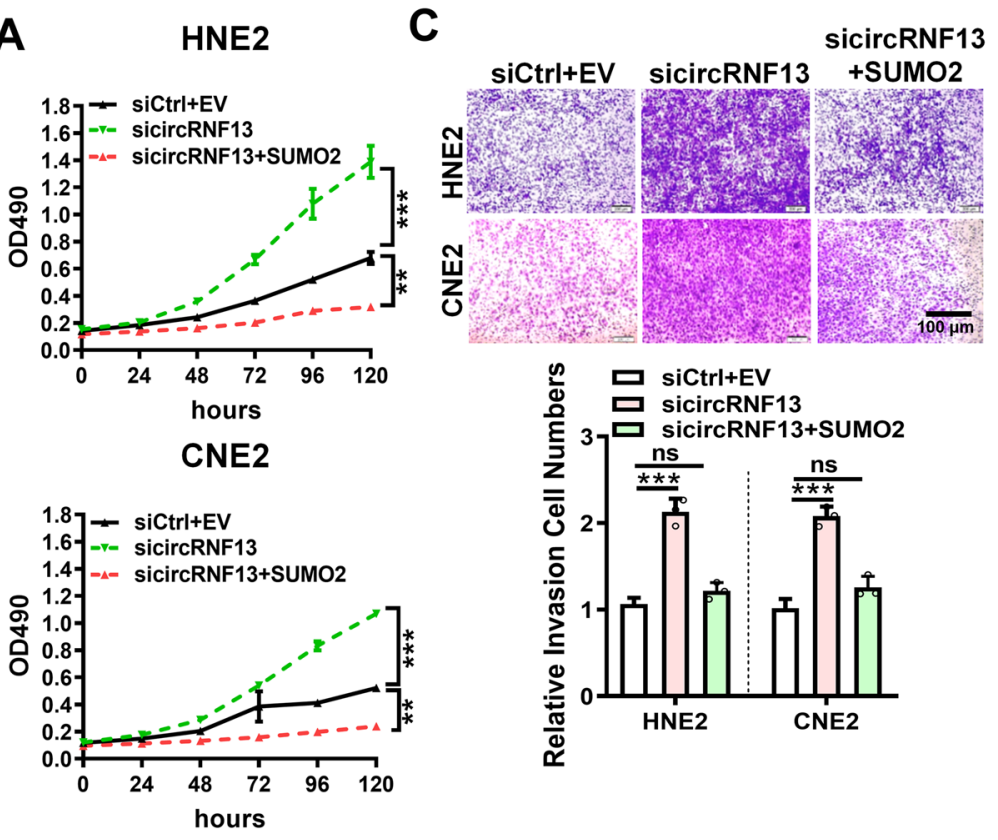

B
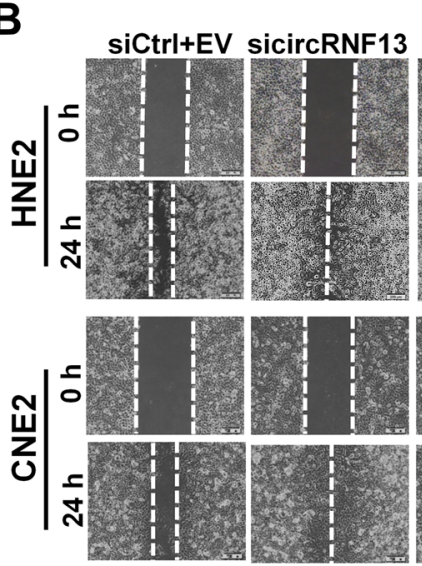

E
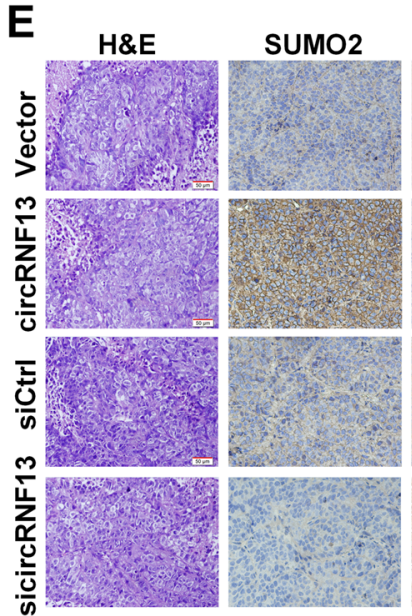

sicircRNF13
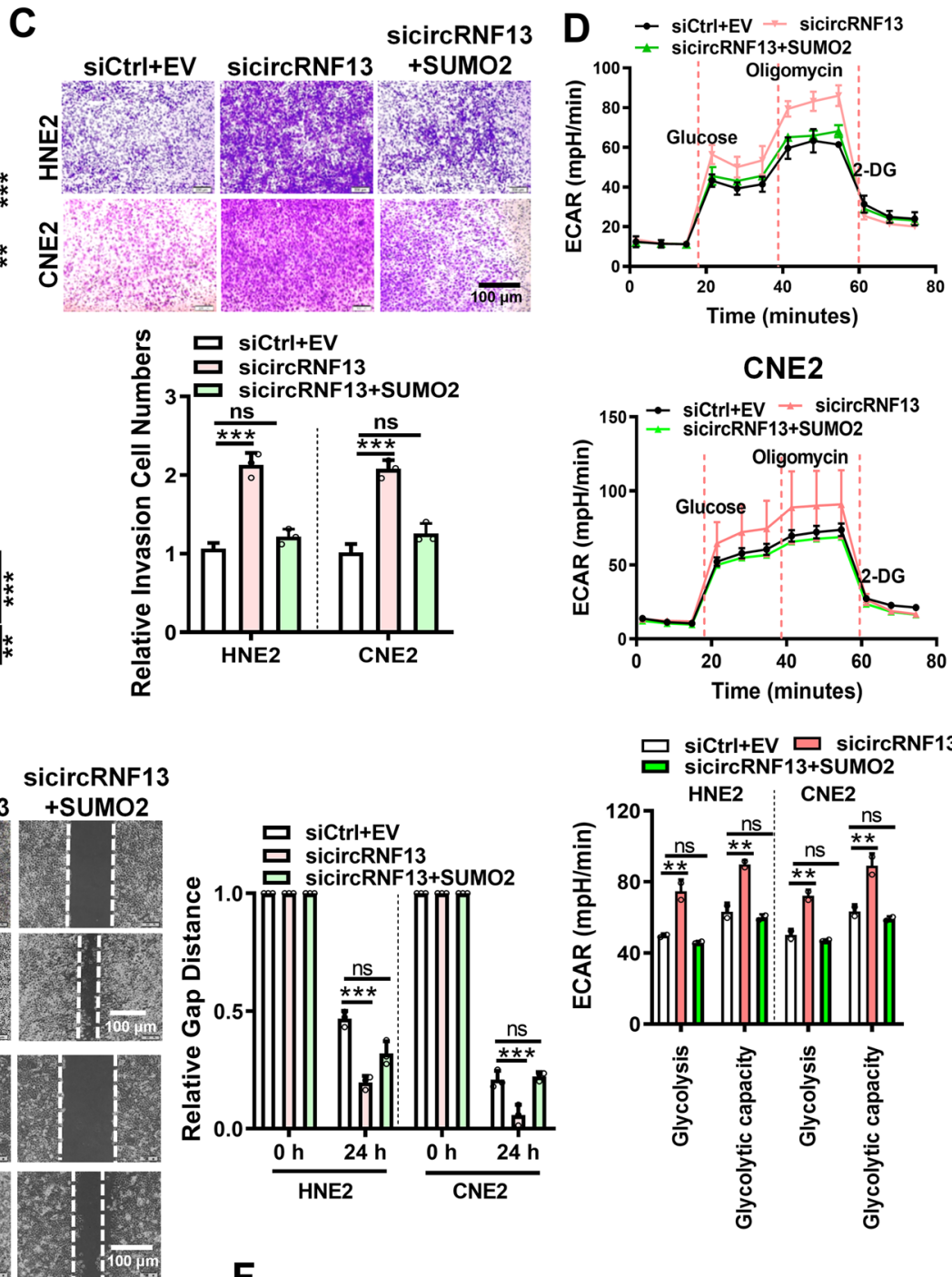

\section{CNE2}

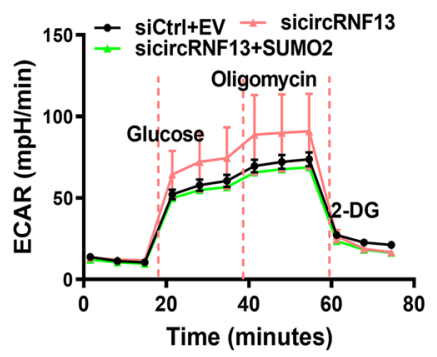

$\square$ siCtrl+EV $\square$ sicircRNF13 sicircRNF13+SUMO2

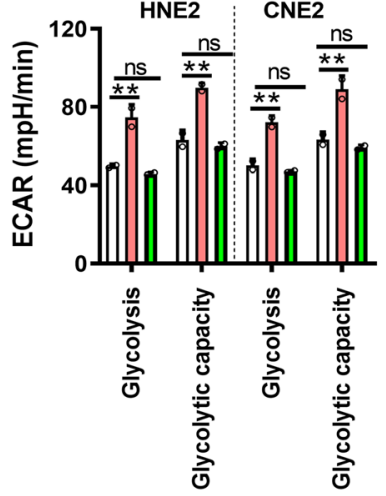

$\mathbf{F}$
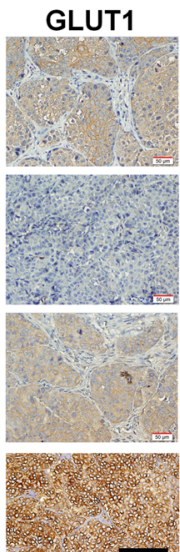

$50 \mu i m$

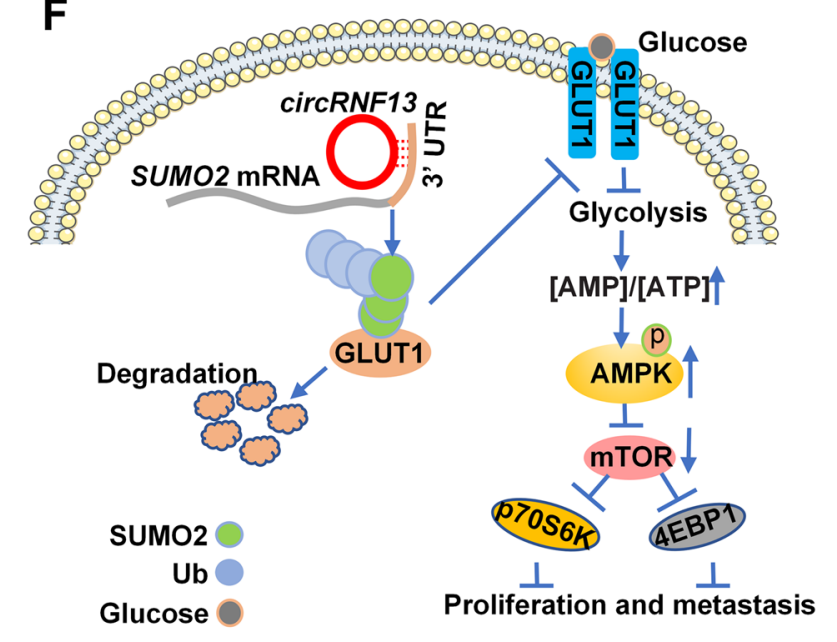

Fig. 8 (See legend on previous page.) 
GLUT1 is the most widely distributed glucose transporter protein [34]. High expression of GLUT1 has been reported in various tumors, such as liver, gastric, and breast cancers [35-38]. While the transcriptional regulation of GLUT1 has been well studied, the posttranslational modifications of GLUT1 have relatively few reports, especially ubiquitination modifications of GLUT1. Xu et al. found that SALL4 recruits the E3 ubiquitin ligase CUL4B to GLUT1, which reduces the expression levels of GLUT1, and subsequently, inhibits glycolysis in cancer cells [39]. Lin et al. found that the E3 ubiquitin ligase family SCF complex Skp2 reduces GLUT1 expression and inhibits glycolysis in tumor cells by ubiquitinating Akt [40]. In this study, we found that SUMO2 directly promotes the degradation of GLUT1 by means of SUMOylation and ubiquitination. This further increases the understanding of the regulatory mechanism of GLUT1. More importantly, blocking the nutritional source of cells by targeting GLUT1 is an important strategy for the development of antitumor drugs. Study of GLUT1 ubiquitination and SUMOylation will likely provide new ideas for drug development.

RNF13 is an E3 ubiquitin-protein ligase with a structural domain of a typical RING zinc-finger protein. In this study, we determined that circRNF13 is a circular splicing of exons 2-8 of the RNF13 gene. Our study demonstrated that circRNF13 is expressed at low levels in NPC and inhibits its proliferation and metastasis. The expression and function of circRNF13 are inconsistent with those of its parental gene RNF13, as the latter exerts an oncogenic function. Overexpression of the RNF13 enzyme is apparent in various human cancers, including basal cell carcinoma, melanoma, and ovarian carcinoma [41, 42]. RNF13, which acts as a ubiquitin ligase, participates in cancer invasion and metastasis. In this study, we analyzed the expression of RNF13 in the online GEO NPC database and our clinical NPC samples. There was no difference in RNF13 expression between the NPE and NPC clinical samples. Knockdown of RNF13 also had no effect on the ubiquitination of GLUT1. RNF13 mRNA could not bind to SUMO2 mRNA in the RNA pull-down experiment (data not shown). These results suggested that the function of circRNF13 is independent of RNF13 mRNA, in promoting ubiquitination and SUMOylation of GLUT1, by binding to SUMO2 mRNA.

\section{Conclusions}

Our results revealed that the novel circRNF13 plays an important role in the development of NPC through the circRNF13-SUMO2-GLUT1 axis. This study implies that circRNF13 mediates glycolysis in NPC by binding to SUMO2 and provides an important theoretical basis for further elucidating the pathogenesis of NPC and targeted therapy.

\section{Abbreviations}

NPC: Nasopharyngeal carcinoma; circRNA: Circular RNAs; RNF13: Ring Finger Protein 13; SUMO2: Small Ubiquitin-like Modifier 2; LC-MS/MS: Liquid Chromatography-tandem Mass Spectrometry; IPA: Ingenuity Pathway Analysis; UTR: Untranslated Region; UBC9: Ubiquitin-Conjugating Enzyme 9; GLUT1: Glucose transporter type 1; LDHA: Lactate Dehydrogenase A; HK2: Hexokinase 2.

\section{Supplementary Information}

The online version contains supplementary material available at https://doi. org/10.1186/s12943-021-01409-4.

Additional file 1: Suppemental Table 1. Probes for fluorescence in situ hybridization and RNA pulldown, siRNAs, primers for RT-PCR and vectors. Suppemental Table 2. List of antibodies for immunohistochemistry, western blotting, immunofluorescence, and RNA pulldown experiments Suppemental Table 3. The top 20 circRNAs identified in NPC 5-8F cells using the next-generation sequencing of mRNA (RNA-seq). Suppemental Table 4. Clinicopathological data of 36 NPC and 12 NPE tissues measured by RT-PCR. Suppemental Table 5. Proteomic analysis of the circRNF13regulated proteins in CNE2 cells transfected with sicircRNF13 or scrambled siRNA identified by the LC-MS/ MS spectrometry. Suppemental Table 6. The top 20 of the cirCRNF13-regulated differential proteins in CNE2 cells after knockdown of circRNF13 identified by the LC-MS/MS spectrometry.

Additional file 2: Fig. S1. CircRNF13 is lowly expression in NPC. A The top 20 circRNAs in the RNA-seq data were tested in NPC $(n=36)$ and NPE ( $n$ $=12$ ) tissues using RT-PCR. NPE, non-tumor nasopharyngeal epithelial tissues. Data have been represented as mean \pm standard deviation (SD). ${ }^{*}, p<0.05 ;{ }^{* *}, p<0.01 ;{ }^{* * *}, p<0.0001$. B Expression of circRNF13 was verified using qPCR in NPC cell lines 5-8F, CNE2, HNE2,HONE1, and 6-10B and normal immortal nasopharyngeal epithelial NP69 cells. Fig. S2. CircRNF13 inhibits proliferation, migration, and invasion of NPC. The overexpression or knockdown efficiencies of circRNF13 were measured in HNE2 and CNE2 cells after transfection with circRNF13 overexpression vector or circRNF13 siRNAs. RNF13 mRNA expression was not affected by transfection. All experiments were repeated at least three times. Data have been represented as mean \pm SD. ${ }^{* *}, p<0.001$. Fig. S3. CircRNF13 inhibits glycolysis in NPC cells. A The proteomics profile in CNE2 cells was analyzed upon circRNF13 knockdown. A total of 294 differentially expressed proteins between sicircRNF13 and scrambled siRNAs were screened, including 100 proteins upregulated and 194 proteins downregulated by sicircRNF13. B The glycolysis pathway was enriched according to the 294 differential proteins screened from the LC-MS/MS data, using gene set enrichment analysis with the IPA software. C The extracellular acidification rate (ECAR) in HNE2 and CNE2 cells was measured using Seahorse XF assays, in response to circRNF13 overexpression or knockdown. D Representative images of wound-healing assay showed that the glycolysis inhibitor 2-DG attenuated the effect of circRNF13 on NPC cell migration when HNE2 and CNE2 cells transfected with sicircRNF13 were treated with it. Fig. S4. SUMO2 inhibits proliferation, migration, invasion, and glycolysis in NPC cells. A The overexpression efficiency of SUMO2 was measured in HNE2 and CNE2 cells using RT-PCR and western blotting, after transfection of the SUMO2 overexpression vector. Data have been represented as mean \pm SD. ${ }^{* * *}, p<0.001$. B Overexpression of SUMO2 inhibited proliferation of HNE2 and CNE2 cells, as assessed using MTT assay. All experiments were repeated at least three times. Data have been represented as mean \pm SD. ${ }^{*}, p<0.05 ;{ }^{* *}, p<0.01{ }^{* * *}, p<0.001$. C The migration ability of HNE2 and CNE2 cells was significantly reduced upon overexpression of SUMO2, as assessed using wound-healing assays. All experiments were repeated at least three times. Data have been represented as mean $\pm S D$. ${ }^{* *}, p$ $<0.01 ;{ }^{* * *}, p<0.001$. D The invasive ability of HNE2 and CNE2 cells was significantly decreased upon overexpression of SUMO2, as assessed using Transwell assays. All experiments were repeated at least three times. Data 
have been represented as mean \pm SD. ${ }^{* * *}, p<0.001$. EThe extracellular acidification rate (ECAR) was measured using Seahorse XF assays upon overexpression of SUMO2 in HNE2 and CNE2 cells. Glycolysis, glycolytic capacity, and glycolytic reserve were analyzed. All experiments were repeated at least three times. Data have been represented as mean \pm SD. ${ }_{*}^{*}, p<0.01$. Fig. S5. SUMO2 promotes degradation of GLUT1. A The binding motifs for SUMOylation on the GLUT1 protein (K245 and K451) were predicted using GPS-SUMO 2.0 website. B Expression of GLUT1 protein was examined in HNE2 and CNE2 cells using western blotting, after overexpression of SUMO2. C Degradation of GLUT1 was detected in HNE2 and CNE2 cells using western blotting, after overexpression of SUMO2 and treatment with $50 \mu \mathrm{g} / \mathrm{mL}$ cycloheximide (CHX).

\section{Acknowledgements}

We thank Prof. Minghua Wu for providing HA-SUMO2 overexpression plasmid and Prof. Zheng Li for the help of mass spectrometry.

\section{Authors' contributions}

Wei Xiong and Zhaoyang Zeng conceived and designed the project. Yongzhen Mo and Yumin Wang completed the majority of experiments and analyzed the data. Fang Xiong, Xianjie Jiang, Yian Wang, Chunmei Fan, Le Tang performed some of the experiments. Shuai Zhang, Qijia Yan, Shanshan Zhang, Zhaojian Gong, Qianjin Liao collected tissue samples. Yongzhen Mo and Yumin Wang wrote the manuscript. Fuyan Wang, Guo Can, Yong Li, Xiaoling Li, Guiyuan Li, Wei Xiong, Zhaoyang Zeng revised the manuscript. Wei Xiong and Zhaoyang Zeng are responsible for research supervision and funding acquisition. All authors read and approved the final manuscript.

\section{Funding}

This study was funded by the National Natural Science Foundation of China (U20A20367, 82073135, 82072374, 82002239, 81972776 and 81803025), the Overseas Expertise Introduction Project for Discipline Innovation (111 Project, No. 111-2-12), the Natural Science Foundation of Hunan Province (2020JJ4766, 2020JJ4125 and 2019JJ50872), and the Fundamental Research Funds for the Central South University (2020zzts232).

\section{Availability of data and materials}

All data that support the findings of this study are available from the corresponding authors upon reasonable request.

\section{Declarations}

\section{Ethics approval and consent to participate}

The present study was approved by the Ethics Committee of Central South University.

\section{Consent for publication}

Not applicable.

\section{Competing interests}

The authors declare have no conflict of interest.

\section{Author details}

${ }^{1} \mathrm{NHC}$ Key Laboratory of Carcinogenesis and Hunan Key Laboratory of Cancer Metabolism, Hunan Cancer Hospital and Affiliated Cancer Hospital of Xiangya School of Medicine, Central South University, Changsha 410078, Hunan, China. ${ }^{2}$ Key Laboratory of Carcinogenesis and Cancer Invasion of the Chinese Ministry of Education, Cancer Research Institute, Central South University, Changsha 410078, Hunan, China. ${ }^{3}$ Department of Otolaryngology Head and Neck Surgery, Xiangya Hospital, Central South University, Changsha 410078, Hunan, China. ${ }^{4}$ Department of Stomatology, Xiangya Hospital, Central South University, Changsha 410078, Hunan, China. ${ }^{5}$ Department of Oral and Maxillofacial Surgery, The Second Xiangya Hospital, Central South University, Changsha 410011, Hunan, China. ${ }^{6}$ Department of Medicine, Dan L Duncan Comprehensive Cancer Center, Baylor College of Medicine, Houston, TX, USA.

Received: 7 May 2021 Accepted: 14 August 2021 Published online: 31 August 2021

\section{References}

1. Chen YP, Chan ATC, Le QT, Blanchard P, Sun Y, Ma J. Nasopharyngeal carcinoma. Lancet. 2019;394(10192):64-80.

2. You R, Liu YP, Huang PY, Zou X, Sun R, He YX, et al. Efficacy and safety of locoregional radiotherapy with chemotherapy vs chemotherapy alone in de novo metastatic nasopharyngeal carcinoma: a multicenter phase 3 randomized clinical trial. JAMA Oncol. 2020;6(9):1345-52.

3. Zhao J, Guo C, Xiong F, Yu J, Ge J, Wang H, et al. Single cell RNA-seq reveals the landscape of tumor and infiltrating immune cells in nasopharyngeal carcinoma. Cancer Lett. 2020;477:131-43.

4. Mo Y, Wang Y, Zhang L, Yang L, Zhou M, Li X, et al. The role of Wnt signaling pathway in tumor metabolic reprogramming. J Cancer. 2019;10(16):3789-97.

5. Liu YP, Wen YH, Tang J, Wei Y, You R, Zhu XL, et al. Endoscopic surgery compared with intensity-modulated radiotherapy in resectable locally recurrent nasopharyngeal carcinoma: a multicentre, open-label, randomised, controlled, phase 3 trial. Lancet Oncol. 2021;22(3):381-90.

6. Wang D, Zeng Z, Zhang S, Xiong F, He B, Wu Y, et al. Epstein-Barr virusencoded miR-BART6-3p inhibits cancer cell proliferation through the LOC553103-STMN1 axis. FASEB J. 2020;34(6):8012-27.

7. Zhu K, Ge J, He Y, Li P, Jiang X, Wang J, et al. Bioinformatics Analysis of the Signaling Pathways and Genes of Gossypol Induce Death of Nasopharyngeal Carcinoma Cells. DNA Cell Biol. 2021;40(8):1052-63.

8. Zhong Y, Du Y, Yang X, Mo Y, Fan C, Xiong F, et al. Circular RNAs function as ceRNAs to regulate and control human cancer progression. Mol Cancer. 2018;17(1):79.

9. Wang Y, Mo Y, Gong Z, Yang X, Yang M, Zhang S, et al. Circular RNAs in human cancer. Mol Cancer. 2017;16(1):25.

10. Chen $L L$. The expanding regulatory mechanisms and cellular functions of circular RNAs. Nat Rev Mol Cell Biol. 2020;21(8):475-90.

11. Dai S, Mo Y, Wang Y, Xiang B, Liao Q, Zhou M, et al. Chronic Stress Promotes Cancer Development. Front Oncol. 2020;10:1492.

12. Yang $Y$, Fan $X$, Mao $M$, Song $X$, Wu $P$, Zhang $Y$, et al. Extensive translation of circular RNAs driven by N(6)-methyladenosine. Cell Res. 2017;27(5):626-41.

13. Zhang Y, Xue W, Li X, Zhang J, Chen S, Zhang JL, et al. The Biogenesis of Nascent Circular RNAs. Cell Rep. 2016;15(3):611-24.

14. Wang Y, Mo Y, Peng M, Zhang S, Gong Z, Yan Q, et al. The influence of circular RNAs on autophagy and disease progression. Autophagy. 2021:114. https://doi.org/10.1080/15548627.2021.1917131. Online ahead of print.

15. Li P, Zhu K, Mo Y, Deng X, Jiang X, Shi L, et al. Research Progress of circRNAs in Head and Neck Cancers. Front Oncol. 2021;11.

16. Zheng Q, Bao C, Guo W, Li S, Chen J, Chen B, et al. Circular RNA profiling reveals an abundant circHIPK3 that regulates cell growth by sponging multiple miRNAs. Nat Commun. 2016;7:11215.

17. Li X, Yang L, Chen LL. The Biogenesis, Functions, and Challenges of Circular RNAs. Mol Cell. 2018;71(3):428-42.

18. Kristensen LS, Andersen MS, Stagsted LVW, Ebbesen KK, Hansen TB, Kjems J. The biogenesis, biology and characterization of circular RNAs. Nat Rev Genet. 2019;20(11):675-91.

19. Wu P, Mo Y, Peng M, Tang T, Zhong Y, Deng $X$, et al. Emerging role of tumor-related functional peptides encoded by IncRNA and circRNA. Mol Cancer. 2020;19(1):22.

20. Ma X, Yang T, Luo Y, Wu L, Jiang Y, Song Z, et al. TRIM28 promotes HIV-1 latency by SUMOylating CDK9 and inhibiting P-TEFb. Elife. 2019;8.

21. Aichem A, Sailer C, Ryu S, Catone N, Stankovic-Valentin N, Schmidtke G, et al. The ubiquitin-like modifier FAT10 interferes with SUMO activation. Nat Commun. 2019;10(1):4452.

22. Commander R, Wei C, Sharma A, Mouw JK, Burton LJ, Summerbell E, et al. Subpopulation targeting of pyruvate dehydrogenase and GLUT1 decouples metabolic heterogeneity during collective cancer cell invasion. Nat Commun. 2020;11(1):1533.

23. Wang Y, Zhang X, Wang Z, Hu Q, Wu J, Li Y, et al. LncRNA-p23154 promotes the invasion-metastasis potential of oral squamous cell carcinoma by regulating Glut1-mediated glycolysis. Cancer Lett. 2018;434:172-83.

24. Fan C, Wang J, Tang Y, Zhao J, He S, Xiong F, et al. circMAN1A2 could serve as a novel serum biomarker for malignant tumors. Cancer Sci. 2019;110(7):2180-8. 
25. Tang $L$, Xiong $W$, Zhang $L$, Wang $D$, Wang $Y$, Wu $Y$, et al. circSETD3 regulates MAPRE1 through miR-615-5p and miR-1538 sponges to promote migration and invasion in nasopharyngeal carcinoma. Oncogene. 2021;40(2):307-21.

26. Hong $X$, Liu N, Liang Y, He Q, Yang X, Lei Y, et al. Circular RNA CRIM1 functions as a ceRNA to promote nasopharyngeal carcinoma metastasis and docetaxel chemoresistance through upregulating FOXQ1. Mol Cancer. 2020;19(1):33.

27. Li W, Lu H, Wang H, Ning $X$, Liu Q, Zhang $H$, et al. Circular RNA TGFBR2 acts as a ceRNA to suppress nasopharyngeal carcinoma progression by sponging miR-107. Cancer Lett. 2021;499:301-13.

28. Ke Z, Xie F, Zheng C, Chen D. CircHIPK3 promotes proliferation and invasion in nasopharyngeal carcinoma by abrogating miR-4288-induced ELF3 inhibition. J Cell Physiol. 2019;234(2):1699-706.

29. Fan C, Qu H, Xiong F, Tang Y, Tang T, Zhang L, et al. CircARHGAP12 promotes nasopharyngeal carcinoma migration and invasion via ezrinmediated cytoskeletal remodeling. Cancer Lett. 2021;496:41-56.

30. Kong LM, Liao CG, Zhang Y, Xu J, Li Y, Huang W, et al. A regulatory loop involving miR-22, Sp1, and c-Myc modulates CD147 expression in breast cancer invasion and metastasis. Cancer Res. 2014;74(14):3764-78.

31. Schneiderhan W, Scheler M, Holzmann KH, Marx M, Gschwend JE, Bucholz M, et al. CD147 silencing inhibits lactate transport and reduces malignant potential of pancreatic cancer cells in in vivo and in vitro models. Gut. 2009;58(10):1391-8.

32. Bost F, Kaminski L. The metabolic modulator PGC-1a in cancer. Am J Cancer Res. 2019 Feb 1;9(2):198-211.

33. Jha MK, Ament XH, Yang F, Liu Y, Polydefkis MJ, Pellerin L, et al. Reducing monocarboxylate transporter MCT1 worsens experimental diabetic peripheral neuropathy. Exp Neurol. 2020;333.

34. Ozerlat I. Kidney cancer: targeted therapy of glucose uptake via GLUT1 kills RCC cells. Nat Rev Urol. 2011;8(9):471.

35. Erber J, Steiner JD, Isensee J, Lobbes LA, Toschka A, Beleggia F, et al. Dual Inhibition of GLUT1 and the ATR/CHK1 kinase axis displays synergistic cytotoxicity in kras-mutant cancer cells. Cancer Res. 2019;79(19):4855-68.

36. Zhao Z, Wang L, Bartom E, Marshall S, Rendleman E, Ryan C, et al. beta-Catenin/Tcf7l2-dependent transcriptional regulation of GLUT1 gene expression by Zic family proteins in colon cancer. Sci Adv. 2019;5(7):eaax0698.

37. Wu Q, Ba-Alawi W, Deblois G, Cruickshank J, Duan S, Lima-Fernandes E, et al. GLUT1 inhibition blocks growth of RB1-positive triple negative breast cancer. Nat Commun. 2020;11(1):4205

38. Nagarajan A, Dogra SK, Sun L, Gandotra N, Ho T, Cai G, et al. Paraoxonase 2 Facilitates Pancreatic Cancer Growth and Metastasis by Stimulating GLUT1-Mediated Glucose Transport. Mol Cell. 2017;67(4):685-701 e6.

39. Kim J, Xu S, Xiong L, Yu L, Fu X, Xu Y. SALL4 promotes glycolysis and chromatin remodeling via modulating HP1alpha-Glut1 pathway. Oncogene. 2017;36(46):6472-9.

40. Chan CH, Li CF, Yang WL, Gao Y, Lee SW, Feng Z, et al. The Skp2-SCF E3 ligase regulates Akt ubiquitination, glycolysis, herceptin sensitivity, and tumorigenesis. Cell. 2012;149(5):1098-111.

41. Jin $\mathrm{X}$, Cheng $\mathrm{H}$, Chen J, Zhu D. RNF13: an emerging RING finger ubiquitin ligase important in cell proliferation. FEBS J. 2011;278(1):78-84.

42. Zhang Q, Meng Y, Zhang L, Chen J, Zhu D. RNF13: a novel RINGtype ubiquitin ligase over-expressed in pancreatic cancer. Cell Res. 2009;19(3):348-57.

\section{Publisher's Note}

Springer Nature remains neutral with regard to jurisdictional claims in published maps and institutional affiliations. 